\title{
THE SUBGROUP-SUBFACTOR
}

\author{
VIJAY KODIYALAM and V. S. SUNDER
}

\begin{abstract}
In this paper, we compute the standard invariant of the 'subgroup-subfactor' $P \times_{\left.\alpha\right|_{H}} H \subset P \times_{\alpha} G$, where $\alpha$ denotes an outer action of a finite group $G$ on a $I I_{1}$ factor $P$, and $P \times_{\left.\alpha\right|_{H}} H$ denotes the obvious crossed-product obtained by restricting the action to $H$. We then use this description to exhibit a pair of non-isomorphic subgroups $H_{i}, i=1,2$, of the symmetric group $S_{4}$ such that the subfactors $P \times_{\left.\alpha\right|_{H_{i}}} H_{i} \subset P \times_{\alpha} G, i=1,2$ are conjugate, thereby disproving a conjecture of Thomsen - see [9] - that 'the subgroup-subfactor remembers the subgroup' (provided the subgroup contains no non-trivial normal subgroup of the ambient group).
\end{abstract}

\section{Introduction}

In this paper, we give a complete and explicit description of the so-called standard invariant - see [7] - of the subgroup-subfactor. There does exist some information in the literature on the principal and dual graphs (for instance, see [4] and [2]) as well as on the fusion rules for the concerned bimodules (see [3]). However, in order to obtain the entire standard invariant, we also need the connection (in the sense of [5]) or the canonical commuting square (in the sense of [7]).

In order to obtain the connection, we need the bimodules in a sufficiently explicit form so that we can write down 'bases of intertwiners', which are the building blocks for the connection. Such computations are somewhat easy when one has a standard model for the bimodules concerned; by this, we mean the following:

Suppose $Q_{1}, Q_{2}$ are $I I_{1}$ factors and $\mathscr{H}$ is a $Q_{1}-Q_{2}$ bimodule which has finite left $Q_{1}$ - and right $Q_{2}$-dimensions which are positive integers. (We shall only encounter such bimodules in the context at hand, so we shall not bother to talk in full generality here about possibly non-integral dimensions.) Suppose, to be specific, that $\operatorname{dim}_{Q_{1},-} \mathscr{H}=d \in \mathrm{N}$. Then there exists a (necessarily faithful) unital normal *-homomorphism $\theta: Q_{2} \rightarrow M_{d}\left(Q_{1}\right)$ such that the bimodule $\mathscr{H}$ is isomorphic to the following 'standard model' $\mathscr{H}_{\theta}$ of a bimodule: as a Hilbert space $\mathscr{H}_{\theta}$ is isomorphic to a direct sum of $d$ copies of $L^{2}\left(Q_{1}\right)$; we shall find it convenient to identify $\mathscr{H}_{\theta}$ with $M_{1 \times d}\left(L^{2}\left(Q_{1}\right)\right.$ ) (and to

Received July 14, 1997. 
think of elements of $\mathscr{H}_{\theta}$ as row-vectors $\xi=\left[\xi_{1} \cdots \xi_{d}\right]$ with entries from $L^{2}\left(Q_{1}\right)$ ); the $Q_{1}-Q_{2}$ bimodule structure is given by matrix-multiplication thus:

$$
\left(x_{1} \cdot \xi \cdot x_{2}\right)_{j}=x_{1} \cdot \xi_{i} \cdot \theta\left(x_{2}\right)_{j}^{i} .
$$

In the foregoing equation, we use the natural $Q_{1}-Q_{1}$ bimodule structure on $L^{2}\left(Q_{1}\right)$ on the right side, and write $a_{j}^{i}$ for the entry in the $i$-th row and $j$-th column of a matrix $A$; also, we have adopted the 'summation convention' (of summing over indices which appear once as a superscript and once as a subscript), and we shall continue to use this convention throughout this paper.

We call $\mathscr{H}_{\theta}$ a 'standard model' for the $Q_{1}-Q_{2}$ bimodule $\mathscr{H}$; the understanding is that its elements are row-vectors with entries coming from $L^{2}\left(Q_{1}\right)$, the left action is by the standard diagonal action, and the right action is transferred from the natural right action of $M_{d}\left(Q_{1}\right)$ via the homomorphism $\theta$. With the foregoing notation, we shall write $d_{\theta}=d=$ $\operatorname{dim}_{Q_{1},-} \mathscr{H}_{\theta}$.

One reason that working with 'standard models' is convenient, is the following simple fact - see [2], for instance:

Suppose $\mathscr{H}_{\theta_{1}}$ and $\mathscr{H}_{\theta_{2}}$ are standard models of two $Q_{1}-Q_{2}$ bimodules, with $d_{\theta_{i}}=d_{i}, i=1,2$. Then every $Q_{1}-Q_{2}$ linear map $\tilde{T}: \mathscr{H}_{\theta_{1}} \rightarrow \mathscr{H}_{\theta_{2}}$ has the form

$$
(\tilde{T} \xi)_{j}=\xi_{i} \cdot t_{j}^{i}
$$

where the matrix $T=\left(\left(t_{j}^{i}\right)\right) \in M_{d_{1} \times d_{2}}\left(Q_{1}\right)$ satisfies

$$
\theta_{1}(q) T=T \theta_{2}(q) \quad \forall q \in Q_{2} .
$$

(Clearly, the matrix equation (1.2) is short-hand for the system

$$
\left(\theta_{1}(q)\right)_{k}^{i} t_{j}^{k}=t_{k}^{i}\left(\theta_{2}(q)\right)_{j}^{k} \quad \forall i, j
$$

of equations. Also, rather than the clumsy use of parentheses above, we shall, in the future, write $\theta_{j}^{i}(q)$ rather than $(\theta(q))_{j}^{i}$, when given a $\theta: Q \rightarrow M_{d}(P)$.)

Conversely, any matrix $T \in M_{d_{1} \times d_{2}}\left(Q_{1}\right)$ which satisfies equation (1.2) defines a unique $Q_{1}-Q_{2}$ linear map $\tilde{T}$ via equation (1.1).

In $\S 2$, we begin by writing down some explicit 'standard models' of irreducible bimodules of the four kinds $-N-N, N-M, M-N, M-M-$ where $N=P \times H$ and $M=P \times G$, and $P$ is an arbitrary $I I_{1}$ factor.

In $\S 3$, we compute the fusion rules governing the system of bimodules described in $\S 2$.

In $\S 4$, we enumerate all the possible intertwiners for our system of bimo- 
dules; we then deduce the principal and dual graphs of $N \subset M$. (We show that the system of bimodules discussed in $\S 2$ is a complete system of representatives of the different irreducible bimodules which appear in the description of these graphs.)

In $\S 5$, we determine the connection of the subgroup-subfactor using the bimodules of $\S 2$ and the 'bases of intertwiners' obtained in $\S 4$, thereby completing the description of the standard invariant.

In $\S 6$, we use the general description obtained in the earlier sections to show that if $H$ and $K$ are the subgroups of the symmetric group $S_{4}$ defined by

$$
H=\{(1),(1234),(13)(24),(1432)\}\left(\cong \mathrm{Z}_{4}\right)
$$

and

$$
K=\{(1),(12),(34),(12)(34)\}\left(\cong \mathrm{Z}_{2} \times \mathrm{Z}_{2}\right),
$$

then the hyperfinite subfactors $R \times H \subset R \times S_{4}$ and $R \times K \subset R \times S_{4}$ have the same standard invariant and are consequently isomorphic (by [6] and [8]).

It is a fact that if $H$ is a subgroup of a finite group $G$ and if there exists a normal subgroup $L$ of $G$ which is contained in $H$, then the subfactors $(R \times H \subset R \times G)$ and $(R \times H / L \subset R \times G / L)$ are isomorphic.

Motivated by a search for a 'relative Dye's theorem', Klaus Thomsen asked the following question - in [9]:

Suppose $H_{i} \subset G_{i}, i=1,2$ are a pair of inclusions of finite groups, such that for each $i=1,2$, the subgroup $H_{i}$ contains no non-trivial normal subgroup of $G_{i}$; suppose, further, that the subfactors $\left(R \times H_{i} \subset R \times G_{i}\right), i=1,2$, are isomorphic (meaning, of course that there exists an isomorphism of $R \times G_{1}$ onto $R \times G_{2}$, which maps $R \times H_{1}$ onto $R \times H_{2}$ ). Does it then follow that there is a group isomorphism of $G_{1}$ onto $G_{2}$ which maps $H_{1}$ onto $H_{2}$ ?

It should be noted that the answer to the above question is affirmative in the extreme case when $H_{i}=\{1\}$.

Since both the subgroups $H$ and $K$ displayed above, have the property that they contain no non-trivial normal subgroup of $S_{4}$; thus, we have answered Thomsen's question in the negative.

\section{The bimodules}

Throughout this paper, we write $M=P \times_{\alpha} G$ and $N=P \times_{\left.\alpha\right|_{H}} H$, where $\alpha$ is an outer action of a finite group $G$ on a $I I_{1}$ factor $P, H$ is a subgroup of $G$, and $\left.\alpha\right|_{H}$ denotes the restriction of $\alpha$ to $H$. Recall that the typical element of $N$ (resp., $M$ ) has the form $\sum_{h \in H} x_{h} h$ (resp., $\sum_{g \in G} x_{g} g$ ) where $x_{h}, x_{g} \in P$, and 
that the product is given by $(x g)\left(y g^{\prime}\right)=x \alpha_{g}(y) g g^{\prime}$, and the adjoint is given by $(x g)^{*}=g^{-1} x^{*}=\alpha_{g^{-1}}\left(x^{*}\right) g^{-1}$, whenever $x, y \in P, g, g^{\prime} \in G$.)

We shall also use the following notation: suppose $G=\coprod_{t=1}^{n} H g_{t} H$ is the decomposition of $G$ into $H$-double cosets where the system $\left\{g_{t}: 1 \leq t \leq n\right\}$ of representatives of the double coset space has been chosen and fixed once and for all; we assume that $g_{1}=1$.

For fixed $t$, we write $H_{t}=H \cap g_{t}^{-1} H g_{t}$. Notice that under the natural action of $H$ on $H \backslash G$ (given by $(h, H g) \mapsto H g h^{-1}$ ), the isotropy subgroup of the right coset $H g_{t}$ is precisely $H_{t}$. Having fixed a $t$, let $H=\coprod_{p=1}^{k_{t}} h_{p}^{(t)} H_{t}$ be the decomposition of $H$ into left $H_{t}$-cosets (with the coset representatives $\left\{h_{p}^{(t)}: 1 \leq p \leq k_{t}\right\}$ having been chosen and fixed once and for all); as before, we assume that $h_{1}^{(t)}=1 \forall t$.

It follows from the definitions that $G=\coprod_{t=1}^{n} \coprod_{p=1}^{k_{t}} H g_{t}\left(h_{p}^{(t)}\right)^{-1}$ is the decomposition of $G$ into right $H$-cosets.

In the sequel, we shall consistently use the notation

$$
I=\left\{(t, p): 1 \leq t \leq n, 1 \leq p \leq k_{t}\right\}
$$

and we shall write

$$
g_{(t, p)}=g_{t}\left(h_{p}^{(t)}\right)^{-1} \quad \forall(t, p) \in I .
$$

In particular, we have $G=\coprod_{r \in I} H g_{r}$. Consequently, we have an action of $G$ on $I$ (corresponding to the natural $G$-action on $H \backslash G$ ) thus:

$$
g \cdot s=r \Leftrightarrow g_{s} g^{-1} g_{r}^{-1} \in H .
$$

Similarly, for each fixed $t$, we have an action of $H$ on $\left\{p: 1 \leq p \leq k_{t}\right\}$ (corresponding to the natural $H$-action on $H / H_{t}$ ), given by

$$
h \cdot q=p \Leftrightarrow\left(h_{p}^{(t)}\right)^{-1} h h_{q}^{(t)} \in H_{t} .
$$

Before proceeding further, we make an observation that we shall have cause to use, viz.

$$
h \cdot(t, q)=(t, h \cdot q) \quad \forall h \in H,(t, q) \in I,
$$

where the left side is defined by equation (2.7), while the right side is defined by equation (2.8). Indeed, suppose $h \cdot(t, q)=r \in I$; then it follows that $g_{r} \in H g_{t} H$, and consequently, we must have $r=(t, p)$ for some $p$; this means that $g_{t}\left(h_{q}^{(t)}\right)^{-1} h^{-1} h_{p}^{(t)} g_{t}^{-1} \in H$, and hence that $\left(h_{q}^{(t)}\right)^{-1} h-1 h_{p}^{(t)} \in H_{t}$; in other words, $h \cdot q=p$, thereby establishing equation (2.9).

In order to simplify notation, if the index $t$ has been fixed, we shall simply write $h_{p}$ for the correct but cumbersome expression $h_{p}^{(t)}$.

For convenience of reference, we single out a fact as a separate lemma; we 
omit the elementary proof, since it is a direct application of the definition of a free action.

LEMMA 1. Let $g \in G$.

(a) The following conditions on an element $x \in M$ are equivalent:

(i) $x y=\alpha_{g}(y) x \forall y \in P$; (ii) there exists a scalar $C \in \mathrm{C}$ such that $x=C g$.

(b) If $x \in N$, then condition (a) (i) is equivalent to the requirement that there exists a scalar $C$ such that $x=1_{H}(g) C g$.

We need one more bit of notation before we can define our bimodules; for $1 \leq t \leq n$, define

$$
H^{t}=H \cap g_{t} H g_{t}^{-1}=\operatorname{ad}\left(g_{t}\right) H_{t},
$$

where we write $a d(g)$ for the inner automorphism of $G$ given by $k \mapsto g k g^{-1}$.

Proposition 2. (a) Fix $1 \leq t \leq n$, and let $\pi: H^{t} \rightarrow U\left(d_{\pi}, \mathrm{C}\right)$ be a unitary representation of $H^{t}$. Then there exists a unique normal *-homomorphism $\tilde{\pi}: N \rightarrow M_{k_{t} d_{\pi}}(N)$ such that

$$
\tilde{\pi}_{q j}^{p i}(x h)=\delta_{p, h \cdot q} \pi_{j}^{i}\left(g_{(t, p)} h g_{(t, q)}^{-1}\right) \alpha_{g_{(t, p)}}(x) g_{(t, p)} h g_{(t, q)}^{-1},
$$

for all $1 \leq p, q \leq k_{t}, 1 \leq i, j \leq d_{\pi}$.

(b) The 'standard model' $\mathscr{H}_{\tilde{\pi}}$ (of an $N-N$ bimodule) is irreducible if and only if the representation $\pi$ of $H^{t}$ is irreducible.

(c) If $\pi_{i}, i=1,2$ are inequivalent irreducible unitary representations of $\mathrm{H}^{t}$, then the bimodules $\mathscr{H}_{\tilde{\pi}_{i}}$ are inequivalent.

Proof. Notice to start with that if $h \cdot q=p$, then (by equation (2.8) and the definition of $H^{t}$ ) we see that the element $g_{t} h_{p}^{-1} h h_{q} g_{t}^{-1} \in H^{t}$ and consequently, the right side of equation (2.10) is a meaningfully defined element of $N$; thus $\tilde{\pi}$ does indeed extend by linearity to a unique map of $N$ into $M_{k_{t} d_{\pi}}(N)$. An easy verification shows that $\tilde{\pi}$ is a normal $*_{\text {-homomorphism. }}$ Hence $\mathscr{H}_{\tilde{\pi}}$ is indeed an $N-N$ bimodule with left-dimension equal to $k_{t} d_{\pi}$, thereby establishing $(a)$.

We dispose of (b) and (c) together, as follows: suppose $\pi_{i}: H^{t_{i}} \rightarrow$ $U\left(d_{\pi_{i}}\right.$, C) is a unitary representation of $H^{t_{i}}$, for $i=1,2$, and suppose $\tilde{T}: \mathscr{H}_{\tilde{\pi}_{1}} \rightarrow \mathscr{H}_{\tilde{\pi}_{2}}$ is an $N-N$ linear mapping. Then, there exist $t_{q j}^{p i} \in N$ such that equations (1.1) and (1.2) are satisfied (with $Q_{1}=Q_{2}=N$ and $\left.\theta_{i}=\tilde{\pi}_{i}, i=1,2\right)$.

Since $\tilde{\pi}_{q j}^{p i}(x)=\delta_{p, q} \delta_{i, j} \alpha_{g_{(t, p)}}(x)$, the requirement $\tilde{\pi}_{1}(x) T=T \tilde{\pi}_{2}(x)$ for all $x \in P$ shows that

$$
\alpha_{g_{\left(t_{1}, p\right)}}(x) t_{q j}^{p i}=t_{q j}^{p i} \alpha_{g_{\left(t_{2}, q\right)}}(x)
$$


for all $p, i, q, j$ and for all $x \in P$.

It follows from Lemma 1(b) that there exist unique scalars $C_{q j}^{p i}$ such that

$$
t_{q j}^{p i}=1_{H}\left(g_{\left(t_{1}, p\right)} g_{\left(t_{2}, q\right)}^{-1}\right) C_{q j}^{p i} g_{\left(t_{1}, p\right)} g_{\left(t_{2}, q\right)}^{-1} .
$$

However $H g_{r} \neq H g_{s}$ for distinct $r, s \in I$; first deduce from this that the bimodules $\mathscr{H}_{\tilde{\pi}_{i}}$ are disjoint (in the sense of not admitting any non-zero $N-N$ linear map between them) if $t_{1} \neq t_{2}$. Thus, we may assume without loss of generality that $t_{1}=t_{2}=t$ (say) in the rest of this proof.

Next, deduce from equation (2.11) that

$$
t_{q j}^{p i}=\delta_{q}^{p}\left(C_{q}\right)_{j}^{i},
$$

for appropriate scalars $\left(C_{q}\right)_{j}^{i}$. (We may and do think of the $C_{q}$ 's as scalar $d_{\pi_{1}} \times d_{\pi_{2}}$ matrices.)

Using the defining equation

$$
\tilde{\pi}_{q j}^{p i}(h)=\delta_{p, h \cdot q} \pi_{j}^{i}\left(g_{(t, p)} h g_{(t, q)}^{-1}\right) g_{(t, p)} h g_{(t, q)}^{-1},
$$

and the expression for $t_{q j}^{p i}$ obtained in equation (2.12), and appealing to equation (2.9) at an appropriate place in the simplification, we find that the required equation $T \tilde{\pi}_{2}(h)=\tilde{\pi}_{1}(h) T$ may be re-written in the form of the matrix equations

$$
C_{h \cdot q} \pi_{2}\left(g_{(t, h \cdot q)} h g_{(t, q)}^{-1}\right)=\pi_{1}\left(g_{(t, h \cdot q)} h g_{(t, q)}^{-1}\right) C_{q} \forall t, q, h .
$$

Since $H$ acts transitively on $H / H_{t}$, and since $h \cdot 1=1$ if and only if $h \in H_{t}$, we may deduce that all the $C_{q}$ 's are uniquely determined by $C_{1}$, and that consequently, the set of $N-N$ linear self-maps of $\mathscr{H}_{\tilde{\pi}}$ is in bijective correspondence with the set of those matrices $C\left(=C_{1}\right) \in M_{d_{\pi_{1}} \times d_{\pi_{2}}}(\mathrm{C})$ which satisfy the condition

$$
C \pi_{2}\left(g_{(t, 1)} h g_{(t, 1)}^{-1}\right)=\pi_{1}\left(g_{(t, 1)} h g_{(t, 1)}^{-1}\right) C, \forall h \in H_{t},
$$

or, in other words, since $a d\left(g_{t}\right)\left(H_{t}\right)=H^{t}$,

$$
C \pi_{2}(h)=\pi_{1}(h) C \forall h \in H^{t} .
$$

The truth of the assertions (b) and (c) is seen to follow easily.

The following propositions are proved in an entirely analogous manner; in fact, the proofs are, if anything, simpler than the case just treated.

Proposition 3. (a) Let $\lambda: H \rightarrow U\left(d_{\lambda}\right.$, C) be a unitary representation of $H$. Then there exists a unique normal $*_{-}$homomorphism $\tilde{\lambda}: M \rightarrow M_{[G: H] d_{\lambda}}(N)$ such that 


$$
\tilde{\lambda}_{s l}^{r k}(x g)=\delta_{r, g \cdot s} \lambda_{l}^{k}\left(g_{r} g g_{s}^{-1}\right) \alpha_{g_{r}}(x) g_{r} g g_{s}^{-1},
$$

for all $r, s \in I, 1 \leq k, l \leq d_{\lambda}$.

(b) The 'standard model' $\mathscr{H}_{\hat{\lambda}}$ (of an $N-M$ bimodule) is irreducible if and only if the representation $\lambda$ of $H$ is irreducible.

(c) If $\lambda_{i}, i=1,2$ are inequivalent irreducible unitary representations of $H$, then the bimodules $\mathscr{H}_{\tilde{\lambda}_{i}}$ are inequivalent.

Proposition 4. (a) Let $\rho: H \rightarrow U\left(d_{\rho}, \mathrm{C}\right)$ be a unitary representation of $H$. Then there exists a unique normal ${ }^{*}$-homomorphism $\tilde{\rho}: N \rightarrow M_{d_{\rho}}(M)$ such that

$$
\rho_{n}^{m}(x h)=\rho_{n}^{m}(h) x h,
$$

for all $1 \leq m, n \leq d_{\rho}$.

(b) The 'standard model' $\mathscr{H}_{\tilde{\rho}}$ (of an $M-N$ bimodule) is irreducible if and only if the representation $\rho$ of $H$ is irreducible.

(c) If $\rho_{i}, i=1,2$ are inequivalent irreducible unitary representations of $H$, then the bimodules $\mathscr{H}_{\tilde{\rho}_{i}}$ are inequivalent.

Proposition 5. (a) Let $\sigma: G \rightarrow U\left(d_{\sigma}\right.$, C) be a unitary representation of $G$. Then there exists a unique normal ${ }^{*}$-homomorphism $\tilde{\sigma}: M \rightarrow M_{d_{\sigma}}(M)$ such that

$$
\tilde{\sigma}_{b}^{a}(x g)=\sigma_{b}^{a}(g) x g,
$$

for all $1 \leq a, b \leq d_{\sigma}$.

(b) The 'standard model' $\mathscr{H}_{\tilde{\sigma}}$ (of an $M-M$ bimodule) is irreducible if and only if the representation $\sigma$ of $G$ is irreducible.

(c) If $\sigma_{i}, i=1,2$ are inequivalent irreducible unitary representations of $G$, then the bimodules $\mathscr{H}_{\tilde{\sigma}_{i}}$ are inequivalent.

Remark 6. Observe that, in the notation of the preceding Propositions, we have

$$
\begin{aligned}
d_{\tilde{\pi}} & =\left[H: H_{t}\right] d_{\pi} \\
d_{\tilde{\lambda}} & =[G: H] d_{\lambda} \\
d_{\tilde{\rho}} & =d_{\rho} \\
d_{\tilde{\sigma}} & =d_{\sigma} .
\end{aligned}
$$

\section{The fusion rules}

We recall - see [2], for instance - some notation and facts about tensor products of bimodules.

Suppose $Q_{1}, Q_{2}$ and $Q_{3}$ are $I I_{1}$ factors with separable preduals, and $\mathscr{H}, \mathscr{K}$ 
and $\mathscr{L}$ are separable $Q_{1}-Q_{2}, Q_{2}-Q_{3}$ and $Q_{1}-Q_{3}$ bimodules respectively. We assume that these are bifinite in the sense of having finite left and right dimensions and further assume that these dimensions are all positive integers.

The tensor product of $\mathscr{H} \otimes_{Q_{2}} \mathscr{K}$ is described most easily using standard models, say $\mathscr{H}_{\theta}$ for $\mathscr{H}$ and $\mathscr{H}_{\phi}$ for $\mathscr{K}$ where $\theta: Q_{2} \rightarrow M_{d_{\theta}}\left(Q_{1}\right)$ and $\phi: Q_{3} \rightarrow M_{d_{\phi}}\left(Q_{2}\right)$; then a standard model for $\mathscr{H}_{Q_{2}} \mathscr{K}$ is given by $\mathscr{H}_{\theta \otimes \phi}$, where $\theta \otimes \phi: Q_{3} \rightarrow M_{d_{\theta} d_{\phi}}\left(Q_{1}\right)$ is defined by: $(\theta \otimes \phi)_{j l}^{i k}\left(x_{3}\right)=\theta_{j}^{i}\left(\phi_{l}^{k}\left(x_{3}\right)\right)$.

By $\left\langle\mathscr{H} \otimes_{Q_{2}} \mathscr{K}, \mathscr{L}\right\rangle$ we denote the (necessarily finite) dimension of the space of $Q_{1}-Q_{3}$ linear maps from $\mathscr{L}$ to $\mathscr{H} \otimes_{Q_{2}} \mathscr{K}$; of course, when $\mathscr{L}$ is irreducible, this number is just the multiplicity with which $\mathscr{L}$ features in the irreducible decomposition of $\mathscr{H} \otimes_{Q_{2}} \mathscr{K}$. By Frobenius reciprocity, calculating the fusion rules between the four kinds of bimodules defined in Section 2 is reduced to calculating $\left\langle\mathscr{H} \otimes_{Q_{2}} \mathscr{K}, \mathscr{L}\right\rangle$ when $\mathscr{H}$ is either an $M-M$ bimodule or an $N-N$ bimodule.

In order to derive the fusion rules for $N-N$ bimodules, it will be convenient to analyse and relate two group actions.

One is an action of $H^{t_{2}}$ on $\left\{p_{1}: 1 \leq p_{1} \leq k_{t_{1}}\right\}$ obtained by restricting the natural action of $H$ on this set.

The other action is that of $H$ on a product of the form $\left\{p_{1}: 1 \leq p_{1} \leq k_{t_{1}}\right\} \times\left\{p_{2}: 1 \leq p_{2} \leq k_{t_{2}}\right\}$ for fixed $t_{1}$ and $t_{2}$; denoting $\left(p_{1}, p_{2}\right)$ by $\underline{p}$, this action is defined by

$$
h \cdot \underline{q}=\underline{p} \Leftrightarrow\left\{\begin{array}{l}
h_{p_{2}}^{-1} h h_{q_{2}} \in H_{t_{2}} \text { and } \\
h_{p_{1}}^{-1} g_{\left(t_{2}, p_{2}\right)} h g_{\left(t_{2}, q_{2}\right)}^{-1} h_{q_{1}} \in H_{t_{1}} .
\end{array}\right.
$$

It is easily verified that equation (3.20) does define an $H$-action.

The facts that we will use about these actions are contained in the following lemma.

Lemma 7. (a) The orbits of the $H^{t_{2}}$-action on $\left\{p_{1}: 1 \leq p_{1} \leq k_{t_{1}}\right\}$ are in bijective correspondence with those of the $H$-action described by equation (3.20). A bijection is given by $\left[q_{1}\right] \mapsto\left[\left(q_{1}, 1\right)\right]$ where [.] stands for "orbit of".

(b) If $g_{\left(t_{1}, q_{1}\right)} g_{\left(t_{2}, q_{2}\right)} \in H g_{\left(t_{3}, q_{3}\right)}$ and $h \cdot \underline{q}=\underline{p}$, then $g_{\left(t_{1}, p_{1}\right)} g_{\left(t_{2}, p_{2}\right)} \in H g_{\left(t_{3}, p_{3}\right)}$ for $p_{3}=h \cdot q_{3}$. In particular, $t_{3}$ depends only on the H-orbit of $\underline{q}$.

Proof. (a) The map $\left[q_{1}\right] \mapsto\left[\left(q_{1}, 1\right)\right]$ is easily seen to be well-defined and injective. Surjectivity follows from the formula $\left(q_{1}, q_{2}\right)=h_{q_{2}} \cdot\left(q_{1}, 1\right)$.

(b) Calculation shows that $g_{\left(t_{1}, p_{1}\right)} g_{\left(t_{2}, p_{2}\right)} g_{\left(t_{3}, p_{3}\right)}^{-1}=h g_{\left(t_{1}, q_{1}\right)} g_{\left(t_{2}, q_{2}\right)} g_{\left(t_{3}, q_{3}\right)}^{-1} h^{\prime}$ with $h=\operatorname{ad}\left(g_{t_{1}}\right)\left(h_{p_{1}}^{-1} g_{\left(t_{2}, p_{2}\right)} h g_{\left(t_{2}, q_{2}\right)}^{-1} h_{q_{1}}\right) \in H$ and $h^{\prime}=\operatorname{ad}\left(g_{t_{3}}\right)\left(h_{q_{3}} h^{-1} h_{p_{3}}\right) \in H$.

For a group $K$, we use the symbol $\hat{K}$ to denote a complete set of inequivalent, irreducible, unitary representations of $K$. 
Proposition 8. Fix $t_{1}, t_{2}$ such that $1 \leq t_{i} \leq n$, and let $\pi_{i} \in \hat{H}^{t_{i}}$. Then,

$$
\begin{aligned}
& \mathscr{H}_{\tilde{\pi}_{1}} \otimes_{N} \mathscr{H}_{\tilde{\pi}_{2}} \cong \\
& \bigoplus_{q_{1}} \bigoplus_{\pi_{3}}\left\langle\left.\left.\pi_{1} \circ \operatorname{ad}\left(g_{\left(t_{1}, q_{1}\right)}\right)\right|_{K} \otimes \pi_{2}\right|_{K},\left.\pi_{3} \circ \operatorname{ad}\left(g_{\left(t_{3}, p_{3}\right)} g_{t_{2}}^{-1}\right)\right|_{K}\right\rangle \cdot \mathscr{H}_{\tilde{\pi}_{3}}
\end{aligned}
$$

where the first direct sum is over the representatives $q_{1}$ of the distinct orbits of the $H^{t_{2}}$-action on $\left\{p_{1}: 1 \leq p_{1} \leq k_{t_{1}}\right\}$, the second direct sum is over all $\pi_{3} \in \hat{H}^{t_{3}}$ where $\left(t_{3}, p_{3}\right)$ is determined uniquely by $g_{\left(t_{1}, q_{1}\right)} g_{t_{2}} \in H g_{\left(t_{3}, p_{3}\right)}$, and $K=H^{t_{2}} \cap \operatorname{ad}\left(g_{\left(t_{1}, q_{1}\right)}^{-1}\right) H^{t_{1}}$.

Proof. It suffices to show that: (i) for a fixed $t_{3}$ and $\pi_{3} \in \hat{H}^{t_{3}}$,

$$
\begin{aligned}
& \left\langle\mathscr{H}_{\tilde{\pi}_{1}} \otimes_{N} \mathscr{H}_{\tilde{\pi}_{2}}, \mathscr{H}_{\tilde{\pi}_{3}}\right\rangle \\
& =\sum_{q_{1}}\left\langle\left.\left.\pi_{1} \circ \operatorname{ad}\left(g_{\left(t_{1}, q_{1}\right)}\right)\right|_{K} \otimes \pi_{2}\right|_{K},\left.\pi_{3} \circ \operatorname{ad}\left(g_{\left(t_{3}, p_{3}\right)} g_{t_{2}}^{-1}\right)\right|_{K}\right\rangle
\end{aligned}
$$

where the sum is over the representatives $q_{1}$ of the distinct orbits of the action of $H^{t_{2}}$ on $\left\{p_{1}: 1 \leq p_{1} \leq k_{t_{1}}\right\}$ that satisfy $g_{\left(t_{1}, q_{1}\right)} g_{t_{2}} \in H g_{\left(t_{3}, p_{3}\right)}$ for some $p_{3}$ (this equation also serving to define $p_{3}$ ); and that (ii) the left dimensions of the $N-N$ bimodules on both sides of equation (3.21) are equal.

By definition and equations (1.1) and (1.2) the left hand side of equation (3.22) is the dimension of the solution space of the system

$$
\left(\tilde{\pi}_{3}\right)_{r_{3} k_{3}}^{p_{3} i_{3}}(x h) t_{q_{1} j_{1} q_{2} j_{2}}^{r_{3} k_{3}}=t_{r_{1} k_{1} r_{2} k_{2}}^{p_{3} i_{3}}\left(\tilde{\pi}_{1} \otimes \tilde{\pi}_{2}\right)_{q_{1} j_{1} q_{2} j_{2}}^{r_{1} k_{1} r_{2} k_{2}}(x h) .
$$

These equations are to be solved for the $t_{q_{1} j_{1} q_{2} j_{2}}^{p_{3} i_{3}} \in N$ and are to hold for all $x \in P, h \in H$ and all $p_{3}, i_{3}, q_{1}, j_{1}, q_{2}, j_{2}$ in the appropriate ranges. For notational convenience we will denote $t_{q_{1} j_{1} q_{2} j_{2}}^{p_{3} i_{3}}$ by $t_{q j}^{p_{3} i_{3}}$.

Using Lemma 1 (b) when $h=1$ shows that there exist scalars $C_{q j}^{p_{3} i_{3}}$ such that $t_{q j}^{p_{3} i_{3}}=C_{q \dot{j}}^{p_{3} i_{3}} 1_{H}\left(g_{\left(t_{3}, p_{3}\right)} g_{\left(t_{2}, q_{2}\right)}^{-1} g_{\left(t_{1}, q_{1}\right)}^{-1}\right) g_{\left(t_{3}, p_{3}\right)} g_{\left(t_{2}, q_{2}\right)}^{-1} g_{\left(t_{1}, q_{1}\right)}^{-1}$. By $\dot{C}_{\underline{q j}}^{p_{3} i_{3}}$ denote $C_{q j}^{p_{3} i_{3}} 1_{H}\left(g_{\left(t_{3}, p_{3}\right)} g_{\left(t_{2}, q_{2}\right)}^{-1} g_{\left(t_{1}, q_{1}\right)}^{-1}\right)$.

Substituting this into the system (3.23) for $x=1$ and simplifying, we obtain the matrix equations

$$
\begin{aligned}
& \pi_{3}\left(g_{\left(t_{3}, p_{3}\right)} h g_{\left(t_{3}, r_{3}\right)}^{-1}\right) \dot{C}_{\underline{q}}^{r_{3}} \\
& =\dot{C}_{\underline{r}}^{p_{3}} \sum_{r}\left(\pi_{1}\left(g_{\left(t_{1}, r_{1}\right)} g_{\left(t_{2}, r_{2}\right)} h g_{\left(t_{2}, q_{2}\right)}^{-1} g_{\left(t_{1}, q_{1}\right)}^{-1}\right) \otimes \pi_{2}\left(g_{\left(t_{2}, r_{2}\right)} h g_{\left(t_{2}, q_{2}\right)}^{-1}\right)\right)
\end{aligned}
$$

where $\underline{r}=h \cdot q$ and $r_{3}=h^{-1} \cdot p_{3}$. These are to hold for all $p_{3}, q$ and $h \in H$.

We now observe that for a fixed $\underline{q}$, if $g_{\left(t_{1}, q_{1}\right)} g_{\left(t_{2}, q_{2}\right)} \notin H g_{t_{3}} \bar{H}$, then all $\dot{C}_{q}^{r_{3}}$ vanish. On the other hand, if $g_{\left(t_{1}, q_{1}\right)} \bar{g}_{\left(t_{2}, q_{2}\right)} \in H g_{t_{3}} H$, then exactly one $\dot{C}_{q}^{r_{3}}$ is possibly non-zero and this $r_{3}$ is determined by $g_{\left(t_{1}, q_{1}\right)} g_{\left(t_{2}, q_{2}\right)} \in H g_{\left(t_{3}, r_{3}\right)}$. Fur- 
ther, by Lemma 7 (b), the $H$-orbit of $\underline{q}$ determines which of these cases will hold.

We need to consider only those $q$ for which there is a possibly non-zero $\dot{C}_{q}^{r_{3}}$ and denote this by $\ddot{C}_{q}$.

One more application of Lemma 7 (b) gives the matrix system

$$
\pi_{3}\left(g_{\left(t_{3}, p_{3}\right)} h g_{\left(t_{3}, r_{3}\right)}^{-1}\right) \ddot{C}_{\underline{q}}=\ddot{C}_{\underline{r}}\left(\pi_{1}\left(g_{\left(t_{1}, r_{1}\right)} g_{\left(t_{2}, r_{2}\right)} h g_{\left(t_{2}, q_{2}\right)}^{-1} g_{\left(t_{1}, q_{1}\right)}^{-1}\right) \otimes \pi_{2}\left(g_{\left(t_{2}, r_{2}\right)} h g_{\left(t_{2}, q_{2}\right)}^{-1}\right)\right)
$$

where again, $\underline{r}=h \cdot q$.

Therefore all the $\bar{C}_{\underline{q}}$ for $\underline{q}$ in a single $H$-orbit are determined by any one of them, say, $\ddot{C}_{\left(q_{1}, 1\right)}$. Since the stabilizer in $H$ of $\left(q_{1}, 1\right)$ is easily calculated to be $H_{t_{2}} \cap \operatorname{ad}\left(g_{t_{2}}^{-1} h_{q_{1}}\right) H_{t_{1}}$, the equations that $\ddot{C}_{\left(q_{1}, 1\right)}$ satisfies are

$$
\pi_{3}\left(g_{\left(t_{3}, p_{3}\right)} h g_{\left(t_{3}, p_{3}\right)}^{-1}\right) \ddot{C}_{\left(q_{1}, 1\right)}=\ddot{C}_{\left(q_{1}, 1\right)} \pi_{1}\left(g_{\left(t_{1}, q_{1}\right)} g_{t_{2}} h g_{t_{2}}^{-1} g_{\left(t_{1}, q_{1}\right)}^{-1}\right) \otimes \pi_{2}\left(g_{t_{2}} h g_{t_{2}}^{-1}\right)
$$

for all $h \in H_{t_{2}} \cap \operatorname{ad}\left(g_{t_{2}}^{-1} h_{q_{1}}\right) H_{t_{1}}$. Finally, replacing $h$ by $\operatorname{ad}\left(g_{t_{2}}\right)(h)$, we get that $\ddot{C}_{\left(q_{1}, 1\right)}$ intertwines $\left.\left.\pi_{1} \circ \operatorname{ad}\left(g_{t_{1}} h_{q_{1}}^{-1}\right)\right|_{K} \otimes \pi_{2}\right|_{K}$ and $\left.\pi_{3} \circ \operatorname{ad}\left(g_{\left(t_{3}, p_{3}\right)} g_{t_{2}}^{-1}\right)\right|_{K}$, finishing the proof of equation (3.22).

We next need to verify that the left dimensions of the $N-N$ bimodules on either side of equation (3.21) are equal. On the right side we have

$$
\sum_{q_{1}} \sum_{\pi_{3}}\left\langle\left.\left.\pi_{1} \circ \operatorname{ad}\left(g_{\left(t_{1}, q_{1}\right)}\right)\right|_{K} \otimes \pi_{2}\right|_{K},\left.\pi_{3} \circ \operatorname{ad}\left(g_{\left(t_{3}, p_{3}\right)} g_{t_{2}}^{-1}\right)\right|_{K}\right\rangle k_{t_{3}} d_{\pi_{3}} .
$$

For a fixed $q_{1},\left(t_{3}, p_{3}\right)$ is determined by $g_{\left(t_{1}, q_{1}\right)} g_{t_{2}} \in H g_{\left(t_{3}, p_{3}\right)}$ and the sum over terms with this $q_{1}$ is given by

$$
k_{t_{3}} \sum_{\pi_{3}}\left\langle\left.\left.\pi_{1} \circ \operatorname{ad}\left(g_{\left(t_{1}, q_{1}\right)} g_{t_{2}} g_{\left(t_{3}, p_{3}\right)}^{-1}\right)\right|_{L} \otimes \pi_{2} \circ \operatorname{ad}\left(g_{t_{2}} g_{\left(t_{3}, p_{3}\right)}^{-1}\right)\right|_{L}, \pi_{3} \mid L\right\rangle d_{\pi_{3}}
$$

where $L=\operatorname{ad}\left(g_{\left(t_{3}, p_{3}\right)} g_{t_{2}}^{-1}\right) K$. By Frobenius reciprocity, this is

$$
\begin{aligned}
& k_{t_{3}} \sum_{\pi_{3}}\left\langle\operatorname{Ind}_{L \uparrow H^{t_{3}}}\left\{\left(\left.\left.\pi_{1} \circ \operatorname{ad}\left(g_{\left(t_{1}, q_{1}\right)} g_{t_{2}} g_{\left(t_{3}, p_{3}\right)}^{-1}\right)\right|_{L} \otimes \pi_{2} \circ \operatorname{ad}\left(g_{t_{2}} g_{\left(t_{3}, p_{3}\right)}^{-1}\right)\right|_{L}\right\}, \pi_{3}\right\rangle d_{\pi_{3}}\right. \\
& =k_{t_{3}}\left[H^{t_{3}}: L\right] d_{\pi_{1}} d_{\pi_{2}}=[H: L] d_{\pi_{1}} d_{\pi_{2}} .
\end{aligned}
$$

As $\mathscr{H}_{\tilde{\pi}_{1}} \otimes_{N} \mathscr{H}_{\tilde{\pi}_{2}}$ has left dimension $k_{t_{1}} k_{t_{2}} d_{\pi_{1}} d_{\pi_{2}}$, we are reduced to showing that $k_{t_{1}} k_{t_{2}}=\sum_{q_{1}}[H: L]$. This holds since the left side is the cardinality of $\left\{p_{1}: 1 \leq p_{1} \leq k_{t_{1}}\right\} \times\left\{p_{2}: 1 \leq p_{2} \leq k_{t_{2}}\right\}$ and the right side is the sum of the orders of the $H$-orbits by Lemma 7 (a).

The proofs of the following propositions are omitted. They are similar to the proof above. We just remark that in Proposition 9 the natural action of $G$ on $I$ plays a role. This action being transitive, there is no sum over orbit representatives. 
Proposition 9. Fix $t$ so that $1 \leq t \leq n$ and let $\pi \in \hat{H}^{t}$. Let $\lambda_{1} \in \hat{H}$. Then,

$$
\mathscr{H}_{\tilde{\pi}} \otimes_{N} \mathscr{H}_{\tilde{\lambda}_{1}} \cong \bigoplus_{\lambda_{2}}<\left.\pi \otimes \lambda_{1} \circ \operatorname{ad}\left(g_{t}^{-1}\right)\right|_{H^{t}},\left.\lambda_{2}\right|_{H^{t}}>\cdot \mathscr{H}_{\tilde{\lambda}_{2}}
$$

where the direct sum is over all $\lambda_{2} \in \hat{H}$.

Proposition 10. For $\sigma_{1}, \sigma_{2} \in \hat{G}$ we have

$$
\mathscr{H}_{\tilde{\sigma}_{1}} \otimes_{M} \mathscr{H}_{\tilde{\sigma}_{2}} \cong \bigoplus_{\sigma_{3}}<\sigma_{1} \otimes \sigma_{2}, \sigma_{3}>\cdot \mathscr{H}_{\tilde{\sigma}_{3}}
$$

where the direct sum is over all $\sigma_{3} \in \hat{G}$.

Proposition 11. For $\sigma \in \hat{G}$ and $\rho_{1} \in \hat{H}$ we have

$$
\mathscr{H}_{\tilde{\sigma}} \otimes_{M} \mathscr{H}_{\tilde{\rho}_{1}} \cong \bigoplus_{\rho_{2}}<\left.\sigma\right|_{H} \otimes \rho_{1}, \rho_{2}>\cdot \mathscr{H}_{\tilde{\rho}_{2}}
$$

where the direct sum is over all $\rho_{2} \in \hat{H}$.

We use the fusion rules to identify contragredients of the bimodules of Section 2 . The notation $\bar{\theta}$ for a unitary representation $\theta$ of a group $K$ denotes the contragredient representation (defined by $\bar{\theta}_{j}^{i}(k)=\overline{\theta_{j}^{i}(k)}$ ).

Corollary 12. Let $1 \leq t \leq n$ and $\pi \in \hat{H}^{t}, \lambda, \rho \in \hat{H}$ and $\sigma \in \hat{G}$. Then,

(a) $\overline{\mathscr{H}_{\tilde{\pi}}} \cong \mathscr{H}_{\tilde{\pi}_{*}}$ where $\pi_{*}$ is defined as follows. Consider $t_{*}$ defined by $H g_{t_{*}} H=H g_{t}^{-1} H$ and suppose that $g_{t_{*}}=h g_{t}^{-1} h^{\prime}$. Then, let $\pi_{*} \in H^{t_{*}}$ be $\bar{\pi} \circ \operatorname{ad}\left(g_{t} h^{-1}\right)$.

(b) $\frac{\mathscr{H}_{\tilde{\lambda}}}{2} \mathscr{H}_{\tilde{\rho}}$ where $\rho=\bar{\lambda}$.

(c) $\overline{\mathscr{H}_{\tilde{\rho}}} \cong \mathscr{H}_{\tilde{\lambda}}$ where $\lambda=\bar{\rho}$.

(d) $\overline{\mathscr{H}_{\tilde{\sigma}}} \cong \mathscr{H}_{\tilde{\bar{\sigma}}}$

Proof. We prove (a), the others being, as usual, similar and simpler.

(a) In general, for irreducible $N-N$ bimodules $\mathscr{H}$ and $\mathscr{K}, \mathscr{K} \cong \overline{\mathscr{H}}$ if and only if $\left\langle\mathscr{H} \otimes_{N} \mathscr{K}, L^{2}(N)\right\rangle=1$. For the trivial representation $\pi_{0}$ of $H^{1}=H$, we have $\mathscr{H}_{\tilde{\pi}_{0}} \cong L^{2}(N)$. Let $1 \leq t, t_{*} \leq n$ and $\pi \in \hat{H}^{t}, \pi_{*} \in \hat{H}^{t_{*}}$. It follows from equation 3.22 that for $\left\langle\mathscr{H}_{\tilde{\pi}} \otimes \mathscr{H}_{\tilde{\pi}_{*}}, \mathscr{H}_{\tilde{\pi}_{0}}\right\rangle$ to be non-zero, there should exist a $q$ such that $1 \leq q \leq k_{t}$ and $g_{(t, q)} g_{t_{*}} \in H$. This uniquely determines $t_{*}$ and then $q$. Suppose that $g_{t_{*}}=h g_{t}^{-1} h^{\prime}$. There is only one term in the sum in equation 3.22 which can be simplified to be $\left\langle\pi \circ \operatorname{ad}\left(g_{t} h^{-1}\right) \otimes \pi_{t_{*}},\left.\pi_{0}\right|_{H^{t *}}\right\rangle$. This is 1 exactly when $\pi_{*}=\bar{\pi} \circ \operatorname{ad}\left(g_{t} h^{-1}\right)$.

ExAmPle 13 . Let $G$ be the symmetric group $S_{n}$. For $1 \leq k<n$, we regard $S_{k}$ as the subgroup of $G$ which fixes each of the letters $k+1, \cdots, n$. Let $H=S_{n-1}$ (be the subgroup of permutations fixing $n$ ). Consider the double coset decomposition $G=H g_{1} H \coprod H g_{2} H$ where $g_{1}=(1)$ and $g_{2}=(n-1 n)$. 
Then, $H^{1}=H=H_{1}$ and $H^{2}=S_{n-2}=H_{2}$. In this case the irreducible $N-N$ bimodules of interest are parametrised by $\left\{\pi: \pi \in \hat{S}_{n-1}\right\} \amalg\left\{\psi: \psi \in \hat{S}_{n-2}\right\}$, the irreducible $N-M$ bimodules by $\left\{\lambda: \lambda \in \hat{S}_{n-1}\right\}$, the irreducible $M-N$ bimodules by $\left\{\rho: \rho \in \hat{S}_{n-1}\right\}$ and the irreducible $M-M$ bimodules by $\left\{\sigma: \sigma \in \hat{S}_{n}\right\}$. Calculation using Propositions 8-10 shows that the fusion between these is given as follows.

$$
\begin{gathered}
\mathscr{H}_{\tilde{\pi}_{1}} \otimes_{N} \mathscr{H}_{\tilde{\pi}_{2}} \cong \oplus_{\pi_{3}}\left\langle\pi \otimes \pi_{2}, \pi_{3}\right\rangle \cdot \mathscr{H}_{\tilde{\pi}_{3}} ; \\
\mathscr{H}_{\tilde{\pi}} \otimes_{N} \mathscr{H}_{\tilde{\psi}_{1}} \cong \mathscr{H}_{\tilde{\psi}_{1}} \otimes_{N} \mathscr{H}_{\tilde{\pi}} \cong \oplus_{\psi_{2}}\left\langle\left.\pi\right|_{S_{n-2}} \otimes \psi_{1}, \psi_{2}\right\rangle \cdot \mathscr{H}_{\tilde{\psi}_{2}} ; \\
\mathscr{H}_{\tilde{\psi}_{1}} \otimes_{N} \mathscr{H}_{\tilde{\psi}_{2}} \cong \oplus_{\pi}\left\langle\psi_{1} \otimes \psi_{2},\left.\pi\right|_{S_{n-2}}\right\rangle \cdot \mathscr{H}_{\tilde{\pi}} \\
\oplus \oplus_{\psi}\left\langle\left.\left.\psi_{1}\right|_{S_{n-3}} \otimes \psi_{2}\right|_{S_{n-3}},\left.\pi\right|_{S_{n-3}}\right\rangle \cdot \mathscr{H}_{\tilde{\psi}} \\
\mathscr{H}_{\tilde{\pi}} \otimes_{N} \mathscr{H}_{\tilde{\lambda}_{1}} \cong \oplus_{\lambda_{2}}\left\langle\pi \otimes \lambda_{1}, \lambda_{2}\right\rangle \cdot \mathscr{H}_{\tilde{\lambda}_{2}} ; \\
\mathscr{H}_{\tilde{\psi}} \otimes_{N} \mathscr{H}_{\tilde{\lambda}_{1}} \cong \oplus_{\lambda_{2}}\left\langle\left.\psi \otimes \lambda_{1}\right|_{S_{n-2}},\left.\lambda_{2}\right|_{S_{n-2}}\right\rangle \cdot \mathscr{H}_{\tilde{\tau}_{2}} . \\
\mathscr{H}_{\tilde{\sigma}_{1}} \otimes_{M} \mathscr{H}_{\tilde{\sigma}_{2}} \cong \oplus_{\sigma}\left\langle\sigma_{1} \otimes \sigma_{2}, \sigma\right\rangle \cdot \mathscr{H}_{\tilde{\sigma}} . \\
\mathscr{H}_{\tilde{\sigma}} \otimes_{M} \mathscr{H}_{\tilde{\rho}_{1}} \cong \oplus_{\rho_{2}}\left\langle\left.\sigma\right|_{S_{n-2}} \otimes \rho_{1}, \rho_{2}\right\rangle \cdot \mathscr{H}_{\tilde{\rho}_{2}} .
\end{gathered}
$$

Observe that in this example, the fusion algebra given by the $N-N$ bimodules is commutative; although, in general, there is no reason why this should be the case.

\section{The intertwiners}

We begin this section with a discussion of the possible intertwiners between the four kinds of bimodules described in $\S 2$. (Thus, we use the notations of Propositions 2, 3, 4 and 5.)

We shall find the following bit of notation convenient in the following proposition: if $\mathscr{H}_{\theta}$ is a 'standard model' of a $Q_{1}-Q_{2}$ bimodule, and $\theta$ is presented as a map from $Q_{2}$ into $M_{S}\left(Q_{1}\right)$, where $S$ is a set of cardinality $d_{\theta}$, then we shall write $e^{s}$ for the element of $\mathscr{H}_{\theta}$ which is the row-vector whose only non-zero co-ordinate is the identity 1 of $Q_{1}$ and occurs at the $s$-th place.

Proposition 14. (a) Let $\tilde{C}: \mathscr{H}_{\tilde{\pi}} \rightarrow \mathscr{H}_{\tilde{\lambda}}$ be an $N-N$ linear mapping. Then there exists a unique scalar matrix $C \in M_{d_{\pi} \times d_{\lambda}}(\mathrm{C})$ such that

$$
\pi(h) C=C \lambda(h) \quad \forall h \in H^{t},
$$

and 


$$
\tilde{C}\left(e^{p i}\right)=C_{k}^{i} e^{(t, p) k}
$$

Conversely any scalar matrix $C$ (of the appropriate size) which satisfies equation (4.25) defines a unique $N-N$ linear mapping $\tilde{C}: \mathscr{H}_{\tilde{\pi}} \rightarrow \mathscr{H}_{\tilde{\lambda}}$ via equation (4.26).

Further, if $\tilde{C}_{i}$ and $C_{i}$ are related as above, for $i=1,2$, then the following conditions are equivalent:

(i) $\tilde{C}_{i}^{*} \tilde{C}_{j}=\delta_{i j} \forall 1 \leq i, j \leq 2$, or in words, $\tilde{C}_{1}$ and $\tilde{C}_{2}$ are isometric $N-N$ linear maps with pairwise orthogonal ranges;

(ii) $C_{i} C_{j}^{*}=\delta_{i j} \forall 1 \leq i, j \leq 2$.

(b) Let $\tilde{D}: \mathscr{H}_{\tilde{\lambda}} \rightarrow \mathscr{H}_{\tilde{\sigma}}$ be an $N-M$ linear mapping. Then there exists a unique scalar matrix $D \in M_{d_{\lambda} \times d_{\sigma}}(\mathrm{C})$ such that

$$
\lambda(h) D=D \sigma(h) \forall h \in H,
$$

and

$$
\tilde{D}\left(e^{r k}\right)=\sum_{r \in I} D_{a}^{k} \sigma_{b}^{a}\left(g_{r}\right) g_{r} \cdot e^{b}
$$

Conversely any scalar matrix $D$ (of the appropriate size) which satisfies equation (4.27) defines a unique $N-M$ linear mapping $\tilde{D}: \mathscr{H}_{\tilde{\lambda}} \rightarrow \mathscr{H}_{\tilde{\sigma}}$ via equation (4.28).

Further, if $\tilde{D}_{i}$ and $D_{i}$ are related as above, for $i=1,2$, then the following conditions are equivalent:

(i) $\tilde{D}_{i}{ }^{*} \tilde{D}_{j}=\delta_{i j} \forall 1 \leq i, j \leq 2$, or in words, $\tilde{D}_{1}$ and $\tilde{D}_{2}$ are isometric $N-M$ linear maps with pairwise orthogonal ranges;

(ii) $D_{i} D_{j}^{*}=\delta_{i j} \forall 1 \leq i, j \leq 2$.

(c) Let $\widetilde{E}: \mathscr{H}_{\tilde{\sigma}} \rightarrow \mathscr{H}_{\tilde{\rho}}$ be an $M-N$ linear mapping. Then there exists a unique scalar matrix $E \in M_{d_{\sigma} \times d_{\rho}}(\mathrm{C})$ such that

$$
\sigma(h) E=E \rho(h) \forall h \in H,
$$

and

$$
\tilde{E}\left(e^{a}\right)=E_{m}^{a} e^{m} .
$$

Conversely any scalar matrix $E$ (of the appropriate size) which satisfies equation (4.29) defines a unique $M-N$ linear mapping $\tilde{E}: \mathscr{H}_{\tilde{\sigma}} \rightarrow \mathscr{H}_{\tilde{\rho}}$ via equation (4.30).

Further, if $\tilde{E}_{i}$ and $E_{i}$ are related as above, for $i=1,2$, then the following conditions are equivalent:

(i) $\tilde{E}_{i} \tilde{E}_{j}{ }^{*}=\delta_{i j} \forall 1 \leq i, j \leq 2$, or in words, $\tilde{E}_{1}$ and $\tilde{E}_{2}$ are co-isometric $M-N$ linear maps with pairwise orthogonal initial spaces;

(ii) $E_{i}^{*} E_{j}=\delta_{i j} \forall 1 \leq i, j \leq 2$. 
(d) Let $\tilde{F}: \mathscr{H}_{\tilde{\rho}} \rightarrow \mathscr{H}_{\tilde{\pi}}$ be an $N-N$ linear mapping. Then there exists a unique scalar matrix $F \in M_{d_{\rho} \times d_{\pi}}(\mathrm{C})$ such that

$$
\rho(h) F=F\left(\pi \circ \operatorname{ad}\left(g_{t}\right)\right)(h) \forall h \in H_{t},
$$

and

$$
\tilde{F}\left(g_{r} \cdot e^{m}\right)=\delta_{(t, p)}^{r}\left(\rho\left(h_{p}\right) F\right)_{i}^{m} e^{p i} .
$$

Conversely any scalar matrix $F$ (of the appropriate size) which satisfies equation (4.31) defines a unique $N-N$ linear mapping $\tilde{F}: \mathscr{H}_{\tilde{\rho}} \rightarrow \mathscr{H}_{\tilde{\pi}}$ via equation (4.32).

Further, if $\tilde{F}_{i}$ and $F_{i}$ are related as above, for $i=1,2$, then the following conditions are equivalent:

(i) $\tilde{F}_{i} \tilde{F}_{j}{ }^{*}=\delta_{i j} \forall 1 \leq i, j \leq 2$, or in words, $\tilde{F}_{1}$ and $\tilde{F}_{2}$ are co-isometric $N-N$ linear maps with pairwise orthogonal initial spaces;

(ii) $F_{i}^{*} F_{j}=\delta_{i j} \forall 1 \leq i, j \leq 2$.

Proof. (a) Suppose $\tilde{C}: \mathscr{H}_{\tilde{\pi}} \rightarrow \mathscr{H}_{\tilde{\lambda}}$ is an $N-N$ linear mapping. Then, by equations (1.1) and (1.2), there exists a matrix $T=\left(\left(t_{s l}^{p i}\right)\right) \in$ $M_{\left[H: H_{t}\right] d_{\pi} \times[G: H] d_{\lambda}}(N)$ such that

$$
(\tilde{C} \xi)_{s l}=\xi_{p i} \cdot t_{s l}^{p i}
$$

and

$$
\tilde{\pi}(x h) T=T \tilde{\lambda}(x h) \forall x \in P, h \in H .
$$

Setting $h=1$ in equation (4.33), we find that

$$
\alpha_{g_{(t, p)}}(x) t_{s l}^{p i}=t_{s l}^{p i} \alpha_{g_{s}}(x)
$$

for all $p, i, s, l, x$. Deduce from Lemma 1 that there exist uniquely determined scalars $\gamma_{s l}^{p i}$ such that

$$
t_{s l}^{p i}=\gamma_{s l}^{p i} 1_{H}\left(g_{(t, p)} g_{s}^{-1}\right) g_{(t, p)} g_{s}^{-1} .
$$

Since the $g_{r}$ 's are a system of distinct representatives of the right $H$-cosets, we deduce that

$$
t_{s l}^{p i}=\delta_{s}^{(t, p)} \gamma_{s l}^{p i}
$$

Setting $x=1$ in equation (4.33), and substituting equation (4.34) in the resulting equation, we obtain

$$
\delta_{p, h \cdot q} \delta_{s}^{(t, q)} \gamma_{s l}^{q j} \pi_{j}^{i}\left(g_{(t, p)} h g_{(t, q)}^{-1}\right) g_{(t, p)} h g_{(t, q)}^{-1}=\delta_{r}^{(t, p)} \delta_{h \cdot s}^{r} \gamma_{r k}^{p i} \lambda_{l}^{k}\left(g_{r} h g_{s}^{-1}\right) g_{r} h g_{s}^{-1}
$$

for all $p, i, s, l, h$. 
Notice - thanks to equation (2.9) - that both sides of this equation vanish unless $h \cdot s=(t, p)$, i.e., unless $s=h^{-1} \cdot(t, p)=\left(t, h^{-1} \cdot p\right)$.

Thus, equation (4.35) is seen to be equivalent to the requirement

$$
\begin{aligned}
& \pi_{j}^{i}\left(g_{(t, p)} h g_{\left(t, h^{-1} \cdot p\right)}^{-1}\right) \gamma_{\left(t, h^{-1} \cdot p\right), l}^{h^{-1} \cdot p} g_{(t, p)} h g_{\left(t, h^{-1} \cdot p\right)}^{-1} \\
& =\gamma_{(t, p), k}^{p, i} \lambda_{l}^{k}\left(g_{(t, p)} h g_{\left(t, h^{-1} \cdot p\right)}^{-1}\right) g_{(t, p)} h g_{\left(t, h^{-1} \cdot p\right)}^{-1} .
\end{aligned}
$$

The above equation is to be satisfied for all possible values of $p, i, l, h$. If we introduce the notation

$$
\left(C_{(p)}\right)_{k}^{i}=\gamma_{(t, p), k}^{p, i}
$$

and think of $C_{(p)}$ as a $d_{\pi} \times d_{\lambda}$ scalar matrix, we can re-write equation (4.36) more compactly as

$$
\pi\left(g_{(t, p)} h g_{\left(t, h^{-1} \cdot p\right)}^{-1}\right) C_{\left(h^{-1} \cdot p\right)}=C_{(p)} \lambda\left(g_{(t, p)} h g_{\left(t, h^{-1} \cdot p\right)}^{-1}\right) \forall p, h .
$$

Since $H$ acts transitively on the set of $p$ 's we find that all the $C_{(p)}$ 's are uniquely determined by $C_{(1)}$ via the equation (4.37); and since the isotropy of 1 is the subgroup $H_{t}$, we find that the only condition on $C_{(1)}$ is that

$$
\pi\left(g_{t} h g_{t}^{-1}\right) C_{(1)}=C_{(1)} \lambda\left(g_{t} h g_{t}^{-1}\right) \forall h \in H_{t},
$$

which is precisely the condition (4.25).

Suppose $C_{(1)}$ satisfies the above condition; note that $h_{q} \cdot 1=q$, and that under the specification $p=1, h=h_{q}^{-1}$, the expression $g_{(t, p)} h g_{\left(t, h^{-1} \cdot p\right)}^{-1}$ simplifies to $g_{t} h_{q}^{-1} g_{(t, q)}^{-1}$ which is just 1 , and consequently equation (4.37) reduces, under this specification, to $C_{(q)}=C_{(1)}$; and this is valid for all $q$. Let us simply write $C=C_{(1)}$.

Now deduce from equation (4.34) that

$$
t_{s l}^{p i}=\left\{\begin{array}{l}
C_{l}^{i} \text { if } s=(t, p) \\
0 \text { otherwise }
\end{array}\right.
$$

and consequently,

$$
\begin{aligned}
\tilde{C}\left(e^{p i}\right) & =\left(\left(e^{p i}\right)_{q j} t_{s l}^{q j}\right) \cdot e^{s l} \\
& =C_{l}^{i} e^{(t, p), l}
\end{aligned}
$$

and so the bijective correspondence between the $\tilde{C}$ 's and the $C$ 's has been established.

Suppose, next, that $\tilde{C}_{i}, i=1,2$ satisfy condition (a) (i) of this proposition. It is readily deduced from equation (4.26) that 


$$
\left(\tilde{C}_{i}^{*} e^{s l}\right)=\delta_{(t, p)}^{s}\left(C_{i}^{*}\right)_{j}^{l} e^{p j}, \quad i=1,2, \forall s, l,
$$

where, of course, $\left(C^{*}\right)_{j}^{l}=\overline{C_{l}^{j}}$ denotes the adjoint matrix. Thus, we find that

$$
\begin{aligned}
\delta_{i_{1} i_{2}} \delta^{p_{1}, p_{2}} \delta_{j_{1}, j_{2}} & =\left\langle\tilde{C}_{i_{2}}^{*} \tilde{C}_{i_{1}} e^{p_{1} j_{1}}, e^{p_{2} j_{2}}\right\rangle \\
& =\left\langle\tilde{C}_{i_{1}} e^{p_{1} j_{1}}, \tilde{C}_{i_{2}} e^{p_{2} j_{2}}\right\rangle \\
& =\delta^{p_{1}, p_{2}}\left(C_{i_{1}}\right)^{j_{1} k} \overline{\left(C_{i_{2}}\right)^{j_{2} k}} \\
& =\delta^{p_{1}, p_{2}}\left(C_{i_{1}} C_{i_{2}}^{*}\right)_{j_{2}}^{j_{1}},
\end{aligned}
$$

thereby establishing the validity of (ii).

The implication (ii) $\Rightarrow$ (i) is easy.

The proofs of parts (b)-(d) are entirely similar; we shall say nothing more about those proofs except that in the case of (d), we should note that the $N-M$ bimodule $\mathscr{H}_{\tilde{\rho}}$, when viewed as an $N-N$ bimodule, is the (orthogonal) direct sum of the $N-N$ submodules generated by the vectors $g_{r} \cdot e^{m}, r \in I, 1 \leq m \leq d_{\rho}$.

Now consider the collections $\mathscr{G}(N, N)=\left\{\mathscr{H}_{\tilde{\pi}}: \pi \in \hat{H}^{t}, 1 \leq t \leq n\right\}$ (resp., $\mathscr{G}(N, M)=\left\{\mathscr{H}_{\tilde{\lambda}}: \lambda \in \hat{H}\right\}, \quad$ resp., $\quad \mathscr{G}(M, M)=\left\{\mathscr{H}_{\tilde{\sigma}}: \sigma \in \hat{G}\right\}, \quad$ resp., $\mathscr{G}(M, N)=\left\{\mathscr{H}_{\tilde{\rho}}: \rho \in \hat{H}\right\}$ ) of irreducible $N-N$ (resp., $N-M$, resp., $M-M$, resp., $M-N)$ bimodules.

We list some consequences of Proposition 14 in the following Corollary (where we use the preceding notation).

COROllary 15. (a) The multiplicity with which the $N-N$ bimodule $\mathscr{H}_{\tilde{\pi}}$ is contained in the bimodule $\mathscr{H}_{\tilde{\lambda}}$, when the latter is regarded as an $N-N$ bimodule, is the multpilicity with which the representation $\pi$ features in the representation $\left.\lambda\right|_{H^{t}}$; thus,

$$
\left\langle\mathscr{H}_{\tilde{\lambda}} \otimes_{M M} L^{2}(M)_{N}, \mathscr{H}_{\tilde{\pi}}\right\rangle=\left\langle\operatorname{Res}_{H \downarrow H^{t}} \lambda, \pi\right\rangle .
$$

(b) The multiplicity with which the $N-M$ bimodule $\mathscr{H}_{\tilde{\lambda}}$ is contained in the bimodule $\mathscr{H}_{\tilde{\sigma}}$, when the latter is regarded as an $N-M$ bimodule, is the multpilicity with which the representation $\lambda$ features in the representation $\left.\sigma\right|_{H}$; thus,

$$
\left\langle{ }_{N} L^{2}(M)_{M} \otimes_{M} \mathscr{H}_{\tilde{\sigma}}, \mathscr{H}_{\tilde{\lambda}}\right\rangle=\left\langle\operatorname{Res}_{G \downarrow H} \sigma, \lambda\right\rangle .
$$

(c) The multiplicity with which the $M-N$ bimodule $\mathscr{H}_{\tilde{\rho}}$ is contained in the bimodule $\mathscr{H}_{\tilde{\sigma}}$, when the latter is regarded as an $M-N$ bimodule, is the multpilicity with which the representation $\rho$ features in the representation $\left.\sigma\right|_{H}$; thus,

$$
\left\langle\mathscr{H}_{\tilde{\sigma}} \otimes_{M M} L^{2}(M)_{N}, \mathscr{H}_{\tilde{\rho}}\right\rangle=\left\langle\operatorname{Res}_{G \downarrow H} \sigma, \rho\right\rangle .
$$

(d) The multiplicity with which the $N-N$ bimodule $\mathscr{H}_{\tilde{\pi}}$ is contained in the bimodule $\mathscr{H}_{\tilde{\rho}}$, when the latter is regarded as an $N-N$ bimodule, is the mult- 
pilicity with which the representation $\pi \circ \operatorname{ad}\left(g_{t}\right)$, of the subgroup $H_{t}$, features in the representation $\left.\rho\right|_{H_{t}}$; thus,

$$
\left\langle{ }_{N} L^{2}(M)_{M} \otimes_{M} \mathscr{H}_{\tilde{\rho}}, \mathscr{H}_{\tilde{\pi}}\right\rangle=\left\langle\operatorname{Res}_{H \downarrow H_{t}} \rho, \pi \circ \operatorname{ad}\left(g_{t}\right)\right\rangle .
$$

REMARK 16. (1) If $\pi \cong \bigoplus_{i=1}^{m} \pi_{i}$, where $\pi_{i}, i=1, \cdots, m$ are unitary representations of $H^{t}$, it should be clear that $\mathscr{H}_{\tilde{\pi}}=\oplus_{i=1}^{m} \mathscr{H}_{\tilde{\pi}_{i}}$; and there are three similar remarks corresponding to $\lambda, \sigma$ and $\rho$.

(2) If $\lambda_{0}$ denotes the trivial representation of $H$, it is easily verified that there is a unique $N-M$ linear (norm-preserving) identification of $\mathscr{H}_{\tilde{\lambda}_{0}}$ and $L^{2}(M)$ (regarded as an $N-M$ bimodule). (For $r \in I$, the vector $e^{r}$ corresponds to $g_{r}$ under this identification.)

(3) Observe that Frobenius reciprocity yields equivalent versions of each of the equations (4.38)-(4.41). Thus, for instance, the analogue of (4.41) is

$$
\left\langle{ }_{M} L^{2}(M)_{N} \otimes_{N} \mathscr{H}_{\tilde{\pi}}, \mathscr{H}_{\tilde{\rho}}\right\rangle=\left\langle\operatorname{Ind}_{H_{t} \uparrow H} \pi \circ \operatorname{ad}\left(g_{t}\right), \rho\right\rangle .
$$

Now construct two bipartite graphs by the following specifications: the first graph - call it $\tilde{\Gamma}$ - has even vertices indexed by $\mathscr{G}(N, N)$ and odd vertices indexed by $\mathscr{G}(N, M)$, and the number of bonds that join the even vertex labelled by $\mathscr{H}_{\tilde{\pi}}$, where $\pi \in \hat{H}^{t}$, to the odd vertex labelled by $\mathscr{H}_{\tilde{\lambda}}$, where $\lambda \in \hat{H}$, is given by the common value of the two sides of equation (4.38); while the second graph - call it $\tilde{\Gamma}^{\prime}$ - has even vertices indexed by $\mathscr{G}(M, M)$ and odd vertices indexed by $\mathscr{G}(M, N)$, and the number of bonds that join the even vertex labelled by $\mathscr{H}_{\tilde{\sigma}}$, where $\sigma \in \hat{G}$, to the odd vertex labelled by $\mathscr{H}_{\tilde{\rho}}$, where $\rho \in \hat{H}$, is given by the common value of the two sides of equation (4.40).

Let $\mathscr{B}$ denote the collection of bimodules which index either vertices of $\tilde{\Gamma}$ or vertices of $\tilde{\Gamma}^{\prime}$. Observe the following facts:

(a) It follows from the analysis of $\S 3$ that this collection is 'closed' under formations of tensor-products, when defined, (by which, of course is meant the following: if $Q_{i} \in\{N, M\}, i=1,2,3$, if $\mathscr{H}$ is a $Q_{1}-Q_{2}$ bimodule and $\mathscr{K}$ is a $Q_{2}-Q_{3}$ bimodule such that $\mathscr{H}, \mathscr{K} \in \mathscr{B}$, and if $\mathscr{L}$ is any irreducible $Q_{1}-Q_{3}$ bimodule such that $\left\langle\mathscr{H} \otimes_{Q_{2}} \mathscr{K}, \mathscr{L}\right\rangle \neq 0$, then there exists an $\mathscr{L}_{1} \in \mathscr{B}$ such that $\mathscr{L} \cong \mathscr{L}_{1}$ ).

(b) It also follows from the analysis of $\S 3$ that $\mathscr{B}$ is closed under the formation of contragredients (in the sense of (a) above).

(c) The collection $\mathscr{B}$ contains the irreducible $N-M$ bimodule $L^{2}(M)$.

A moment's reflection on the conditions (a)-(c) above shows that we have proved that the principal and dual graphs, respectively, of the inclusion $N \subset M$ are the connected components $\Gamma$ and $\Gamma^{\prime}$, respectively, of $\tilde{\Gamma}$ and $\tilde{\Gamma}^{\prime}$ which contain $\mathscr{H}_{\tilde{\lambda}_{0}}$ and $\mathscr{H}_{\tilde{\rho}_{0}}$ respectively.

Let $K=\cap_{g \in G} g H^{-1}$ denote the largest normal subgroup of $G$ which is contained in $H$. Observe the following facts: 
(i) $K$ is contained in each $H^{t}$;

(ii) if $G_{1}, G_{2}$ are subgroups of $G$ such that $K \subset G_{1} \subset G_{2}$, and if $\rho$ (resp., $\sigma$ ) is a unitary representation of $G_{1}$ (resp., $G_{2}$ ) which acts trivially on $K$, then so also does $\operatorname{Ind}_{G_{1} \uparrow G_{2}} \rho$ (resp., $\operatorname{Res}_{G_{2} \downarrow G_{1}} \sigma$ ).

(ii) $\lambda_{0}$ (and $\rho_{0}$ ) act trivially on $K$.

It follows easily from (i) - (iii) above that if $\mathscr{B}_{0}$ denotes those bimodules in $\mathscr{B}$ whose corresponding vertex belongs to $\Gamma$ or $\Gamma^{\prime}$, and if $\mathscr{H}_{\tilde{\chi}} \in \mathscr{B}_{0}$, then $\chi$ acts trivially on $K$; in other words, $\chi$, which is 'a priori a representation of some subgroup $G_{0}$ of $G$, may actually be regarded as a unitary representation of $G_{0} / K$.

We now wish to show that all these representations 'occur', meaning:

$$
\mathscr{B}_{0}=\left\{\mathscr{H}_{\tilde{\chi}} \in \mathscr{B}: \chi(k)=i d_{V_{\chi}} \forall k \in K\right\} .
$$

For this, begin by observing that if $\theta$ denotes the permutation representation of $G$ on $I$ (see eq. (2.7)), then $\mathscr{H}_{\tilde{\rho}_{0}} \otimes_{N} \mathscr{H}_{\tilde{\lambda}_{0}} \cong \mathscr{H}_{\theta}$, where $\rho_{0}=\lambda_{0}$ is as in Remark 16 (b). However the representation $\theta$ descends to a faithful and selfcontragredient representation of $G / K$; it follows that every $\sigma \in \hat{G}$ which acts trivially on $K$ will be contained in $\otimes^{n} \theta$ for some $n>0$, and that consequently $\mathscr{H}_{\tilde{\sigma}} \in \mathscr{B}_{0}$, as asserted.

\section{The connection}

Once and for all, fix some choices of irreducible representations of $H^{t}, 1 \leq t \leq n$, of $H$, and of $G$. For all possible $\pi \in \hat{H}^{t}, \lambda, \rho \in \hat{H}$ and $\sigma \in \hat{G}$, define

$$
\begin{aligned}
& m(\pi, \lambda)=\left\langle\operatorname{Res}_{H \downarrow H^{t}} \lambda, \pi\right\rangle \\
& m(\lambda, \sigma)=\left\langle\operatorname{Res}_{G \downarrow H} \sigma, \lambda\right\rangle \\
& m(\rho, \sigma)=\left\langle\operatorname{Res}_{G \downarrow H} \sigma, \rho\right\rangle \\
& m(\pi, \rho)=\left\langle\operatorname{Res}_{H \downarrow H_{t}} \rho, \pi \circ \operatorname{ad}\left(g_{t}\right)\right\rangle,
\end{aligned}
$$

and choose the following collections of matrices with the following properties:

(a) $C_{i}=C_{i}(\pi, \lambda) \in M_{d_{\pi} \times d_{\lambda}}(\mathrm{C}), 1 \leq i \leq m(\pi, \lambda)$ with the property that $\pi(h) C_{i}=C_{i} \lambda(h) \forall h \in H^{t}$ and $C_{i} C_{i^{\prime}}^{*}=\delta_{i i^{\prime}} 1_{d_{\pi}}$ for all $1 \leq i, i^{\prime} \leq m(\pi, \lambda)$ - where $1_{k}$ denotes the $k \times k$ identity matrix; and let $\tilde{C}_{i}$ be related to $C_{i}$ as in Proposition 14 (a), for $1 \leq i \leq m(\pi, \lambda)$.

(b) $D_{j}=D_{j}(\lambda, \sigma) \in M_{d_{\lambda} \times d_{\sigma}}(\mathrm{C}), 1 \leq j \leq m(\lambda, \sigma)$, with the property that $\lambda(h) D_{j}=D_{j} \sigma(h) \forall h \in H$ and $D_{j} D_{j^{\prime}}^{*}=\delta_{j j^{\prime}} 1_{d_{\lambda}}$ for all $1 \leq j, j^{\prime} \leq m(\lambda, \sigma)$; and let $\tilde{D}_{j}$ be related to $D_{j}$ as in Proposition 14 (b), for $1 \leq j \leq m(\lambda, \sigma)$.

(c) $E_{k}=E_{k}(\sigma, \rho) \in M_{d_{\sigma} \times d_{\rho}}(\mathrm{C}), 1 \leq k \leq m(\rho, \sigma)$, with the property that 
$\sigma(h) E_{k}=E_{k} \lambda(h) \forall h \in H$ and $E_{k}^{*} E_{k^{\prime}}=\delta_{k k^{\prime}} 1_{d_{\rho}}$ for all $1 \leq k, k^{\prime} \leq m(\rho, \sigma)$; and let $\tilde{E}_{k}$ be related to $E_{k}$ as in Proposition 14 (c), for $1 \leq k \leq m(\rho, \sigma)$.

(d) $\left.F_{l}=F_{l}(\rho, \pi) \in M_{d_{\rho} \times d_{\pi}}(\mathrm{C}), 1 \leq l \leq m(\pi, \rho)\right)$ with the property that $\rho(h) F_{l}=F_{l}\left(\pi \circ a d\left(g_{t}\right)\right)(h) \forall h \in H_{t} \quad$ and $\quad F_{l}^{*} F_{l^{\prime}}=\delta_{l l^{\prime}} 1_{d_{\pi}}$ for all $1 \leq l, l^{\prime} \leq$ $m(\pi, \rho)$ ); and let $\tilde{F}_{l}$ be related to $F_{l}$ as in Proposition 14 (d), for $1 \leq l \leq m\left(\pi \circ \operatorname{ad}\left(g_{t}\right), \rho\right)$.

Given this data/choice of 'intertwiners', a cell (in the sense of Ocneanu) is (in our specific case) just a collection $\left((\pi, t), C_{i}, \lambda, D_{j}, \sigma, E_{k}, \rho, F_{l}\right)$, where $\pi \in \hat{H}^{t}$ and $1 \leq t \leq n, \lambda, \rho \in \hat{H}, \sigma \in \hat{G}$, and $1 \leq i \leq m(\pi, \lambda), 1 \leq j \leq m(\lambda, \sigma)$, $1 \leq k \leq m(\sigma, \rho)$, and $1 \leq l \leq m(\pi, \rho)$.

Given a cell as above, it is seen that $\tilde{F}_{l} \tilde{E}_{k} \tilde{D}_{j} \tilde{C}_{i}$ is an $N-N$ linear self-map of the irreducible $N-N$ bimodule $\mathscr{H}_{\tilde{\pi}}$, and is consequently a scalar multiple of the identity operator. We shall use the symbol

$$
\begin{array}{ccccc} 
& & C_{i} & & \\
& \lambda & \rightarrow & (\pi, t) & \\
D_{j} & \uparrow & W & \downarrow & F_{l}, \\
& \sigma & \leftarrow & \rho & \\
& & E_{k} & &
\end{array}
$$

to denote the value of the scalar so obtained.

The symbol $W$ is in conformity with Ocneanu's notation for the connection; in fact, the connection, for the subgroup subfactor, is nothing more than the assignment of the number depicted by the expression in (5.43) above to the cell (described by the boundary of this figure). The connection is determined up to the choice of the labelling of the members of $\hat{H}_{t}, \hat{H}$ and $\hat{G}$, and the initial choice of the 'bases of partially isometric intertwiners' $C_{i}, D_{j}, E_{k}, F_{l}$.

For fixed $(\pi, t)$ and $\sigma$, we shall write $W((\pi, t), \sigma)$ to denote the matrix with rows indexed by triples $\left(C_{i}, \lambda, D_{j}\right)$ (where $1 \leq i \leq m(\pi, \lambda), 1 \leq j \leq m(\lambda, \sigma)$ ), and columns indexed by triples $\left(E_{k}, \rho, F_{l}\right)$ (where $1 \leq k \leq m(\sigma, \rho)$, $1 \leq l \leq m(\pi, \rho))$, and whose corresponding entry is given by (5.43). It is a fact (and is one half of the so-called biunitarity condition) that $W((\pi, t), \sigma)$ is a unitary matrix whenever $\left\langle\operatorname{Res}_{G \backslash H^{t}} \sigma, \pi\right\rangle>0$.

The next step is to identify this connection in our context.

Proposition 17. With the foregoing notation, we have

$$
C D \sigma\left(g_{t}\right) E F=\left(\begin{array}{ccccc} 
& & C & & \\
& \lambda & \vec{C} & (\pi, t) & \\
D & \uparrow & W & \downarrow & F \\
& \sigma & \leftarrow & \rho &
\end{array}\right) \cdot 1_{d_{\pi}} .
$$


Proof. Using the notation of Proposition 14, we find that, for all $1 \leq p \leq k_{t}, 1 \leq i \leq d_{\pi}$,

$$
\begin{aligned}
(\tilde{F} \tilde{E} \tilde{D} \tilde{C})\left(e^{p i}\right) & =(\tilde{F} \tilde{E} \tilde{D})\left(C_{k}^{i} e^{(t, p), k}\right) \\
& =C_{k}^{i}(\tilde{F} \tilde{E})\left(D_{a}^{k} \sigma_{b}^{a}\left(g_{(t, p)}\right) g_{(t, p)} \cdot e^{b}\right) \\
& =C_{k}^{i} D_{a}^{k} \sigma_{b}^{a}\left(g_{(t, p)}\right) \tilde{F}\left(g_{(t, p)} \cdot E_{m}^{b} e^{m}\right) \\
& =C_{k}^{i} D_{a}^{k} \sigma_{b}^{a}\left(g_{(t, p)}\right) E_{m}^{b}\left(\rho\left(h_{p}^{(t)}\right) F\right)_{j}^{m} e^{p j} \\
& =\left(C D \sigma\left(g_{(t, p)}\right) E \rho\left(h_{p}^{(t)}\right) F\right)_{j}^{i} e^{p j}
\end{aligned}
$$

Notice, now, that

$$
\begin{aligned}
C D \sigma\left(g_{(t, p)}\right) E \rho\left(h_{p}^{(t)}\right) F & =C D \sigma\left(g_{t}\right) \sigma\left(h_{p}^{(t)}\right)^{-1} E \rho\left(h_{p}^{(t)}\right) F \\
& =C D \sigma\left(g_{t}\right) E F
\end{aligned}
$$

since we aleady know that $(\tilde{F} \tilde{E} \tilde{D} \tilde{C})$ is a scalar operator, this completes the proof; but we can also directly see that the matrix $\left(C D \sigma\left(g_{t}\right) E F\right)$ is a scalar matrix; indeed, to see this, it suffices to check that this matrix commutes with $\pi(h)$ for all $h \in H^{t}$; so pick such an $h$, note that $g_{t}^{-1} h g_{t} \in H_{t} \subset H$, and compute thus, using the intertwining properties of $C, D, E$ and $F$ :

$$
\begin{aligned}
\pi(h)\left(C D \sigma\left(g_{t}\right) E F\right) & =C D \sigma\left(h g_{t}\right) E F \\
& =C D \sigma\left(g_{t}\right) \sigma\left(g_{t}^{-1} h g_{t}\right) E F \\
& =C D \sigma\left(g_{t}\right) E \rho\left(g_{t}^{-1} h g_{t}\right) F \\
& =\left(C D \sigma\left(g_{t}\right) E F\right) \pi(h),
\end{aligned}
$$

as desired.

REMARK 18. Recall - from the end of the last section - that the irreducible representations (of the various groups $G, H, H^{t}$ ) which label the bimodules featuring in $\mathscr{B}_{0}$ are precisely those that act trivially on the subgroup $L=\cap_{g \in G} g \mathrm{Hg}^{-1}$; in other words, these are precisely those irreducible representaions that lie in the images of the canonical embeddings of $\hat{G / L}, \hat{H / L}, H^{t} / L$ in $\hat{G}, \hat{H}, \hat{H}^{t}$. It follows from our description of the connection that the standard invariants of the two subfactors $(P \times H / L \subset P \times G / L)$ and $(P \times H \subset P \times G)$ are naturally identifiable with one another. In particular, we may conclude that

$$
(R \subset R \times G / L) \cong(R \times L \subset R \times G) .
$$

Of course, it is possible to also give a direct proof of this assertion. 


\section{The example}

Throughout this section, the symbol $G$ will denote $S_{4}$ (the symmetric group on the four symbols $1,2,3,4)$, and the symbols $H$ and $K$ will denote the subgroups defined by equations (1.3) and (1.4) respectively. We define

$$
g_{1}=(1), g_{2}=(14)(23), g_{3}=(123),
$$

and note that all the $g_{i}$ 's are even permutations and that $\left\{g_{t}: 1 \leq t \leq 3\right\}$ is simultaneously a system of representatives of the distinct double-cosets of $H$ as well as of $K$; thus,

$$
G=\coprod_{t=1}^{3} H g_{t} H=\coprod_{t=1}^{3} K g_{t} K
$$

In fact, slightly more is true about this choice of representatives; namely, $g_{2}$ normalises $H$ as well as $K$. Thus, imitating the notation we have been using so far and writing

$$
K_{t}=g_{t}^{-1} K g_{t} \cap K, K^{t}=\operatorname{ad}\left(g_{t}\right)\left(K_{t}\right),
$$

we find that

$$
\begin{aligned}
& H_{1}=H_{2}=H \\
& H_{3}=K_{3}=\{1\} \\
& K_{1}=K_{2}=K \\
& H^{1}=H^{2}=H \\
& H^{3}=K^{3}=\{1\} \\
& K^{1}=K^{2}=K .
\end{aligned}
$$

We denote the (1-dimensional) irreducible representations of $H$ by the value they attain on the generator (1234); thus, we write

$$
\hat{H}=\{1, i,-i,-1\},
$$

and the understanding is that $i^{m}\left((1234)^{k}\right)=i^{m k}$. (Naturally, we use the same notation for $\hat{H}^{t}, t=1,2$; and we simply write 1 for the only (trivial) irreducible representation of $H^{3}$.)

We denote the (1-dimensional) irreducible representations of $K$ by ordered pairs (which denote the powers of $(-1)$ which correspond to the values they attain on the generators (12) and (34) respectively; thus, we write

$$
\hat{K}=\{00,10,01,11\},
$$

and the understanding is that $(i j)\left((12)^{m}(34)^{k}\right)=(-1)^{m i+j k}$. (Naturally, we use the same notation for $\hat{K}^{t}, t=1,2$; and for the sake of symmetry, we use the symbol 00 for the only (trivial) irreducible representation of $H^{3}$.) 
Next, we write

$$
\hat{S}_{4}=\{1, \pi, \rho, \epsilon \pi, \epsilon\},
$$

where 1 and $\epsilon$ denote the trivial and alternating (1-dimensional) representations, $\rho$ is 2-dimensional, and $\pi$ and $\epsilon \pi$ are 3-dimensional.

The representation $\epsilon \pi$ is defined by $(\epsilon \pi)(g)=\epsilon(g) \pi(g)$, while we regard $\pi$ as the subrepresentation of the natural permutation representation of $G$ on $\mathrm{C}^{4}$ which is afforded by the orthogonal complement of the constant vector; since all the $g_{i}$ 's are even permutations, we find, thus, that

$$
\begin{aligned}
& \pi\left(g_{1}\right)=\epsilon \pi\left(g_{1}\right)=\left[\begin{array}{llll}
1 & 0 & 0 & 0 \\
0 & 1 & 0 & 0 \\
0 & 0 & 1 & 0 \\
0 & 0 & 0 & 1
\end{array}\right]_{\left.\right|_{V(4) \perp}}, \\
& \pi\left(g_{2}\right)=\epsilon \pi\left(g_{2}\right)=\left[\begin{array}{llll}
0 & 0 & 0 & 1 \\
0 & 0 & 1 & 0 \\
0 & 1 & 0 & 0 \\
1 & 0 & 0 & 0
\end{array}\right]_{\left.\right|_{V(4) \perp}}, \\
& \pi\left(g_{3}\right)=\epsilon \pi\left(g_{3}\right)=\left[\begin{array}{llll}
0 & 0 & 1 & 0 \\
1 & 0 & 0 & 0 \\
0 & 1 & 0 & 0 \\
0 & 0 & 0 & 1
\end{array}\right]_{\left.\right|_{V(4) \perp}},
\end{aligned}
$$

where $V(4)$ is the set of vectors (in $\mathrm{C}^{4}$ ) with all co-ordinates equal.

We shall regard the representation $\rho$ thus: the first thing is to notice that $S_{3}$ is a quotient of $S_{4}$; the convenient way to write the homomorphism $\phi$ of $S_{4}$ onto $S_{3}$ is thus: if $g$ is a transposition, which transposes $i$ and $j$, then $\phi(g)$ is the transposition $(i j)$ or the transposition $(k l)$, where $\{k, l\}=$ $\{1,2,3,4\} \backslash\{i, j\}$, according as $4 \notin\{i, j\}$ or $4 \in\{i, j\}$. As in the case of $\pi$ above, the natural permutation representation of $S_{3}$ on $\mathrm{C}^{3}$ leaves invariant the subspace $V(3)$ of vectors (in $\mathrm{C}^{3}$ ) with all co-ordinates equal; let $\psi$ denote the subrepresentation of this natural representation afforded by the subspace $V(3)^{\perp}$; then $\rho=\psi \circ \phi$. Since

$$
\phi\left(g_{1}\right)=\phi\left(g_{2}\right)=(1), \phi\left(g_{3}\right)=(123),
$$

we thus write: 


$$
\rho\left(g_{1}\right)=\rho\left(g_{2}\right)=\left[\begin{array}{ccc}
c 1 & 0 & 0 \\
0 & 1 & 0 \\
0 & 0 & 1
\end{array}\right]_{\left.\right|_{V(3)^{\perp}}}, \rho\left(g_{3}\right)=\left[\begin{array}{ccc}
0 & 0 & 1 \\
1 & 0 & 0 \\
0 & 1 & 0
\end{array}\right]_{\left.\right|_{V(3)^{\perp}}} .
$$

In the following discussion, we will find it convenient to refer to Figures 1 and 2; so we pause to make some remarks on how these figures are to be interpreted. The subfactor $R \times L \subset R \times G$, where $L$ denotes $H$ or $K$, has finite depth, and is consequently completely determined by the canonical commuting square, viz:

$$
\begin{array}{ccc}
N^{\prime} \cap M_{2 n} & \subset & N^{\prime} \cap M_{2 n+1} \\
\cup & & \cup \\
M^{\prime} \cap M_{2 n} & \subset & M^{\prime} \cap M_{2 n+1}
\end{array}
$$

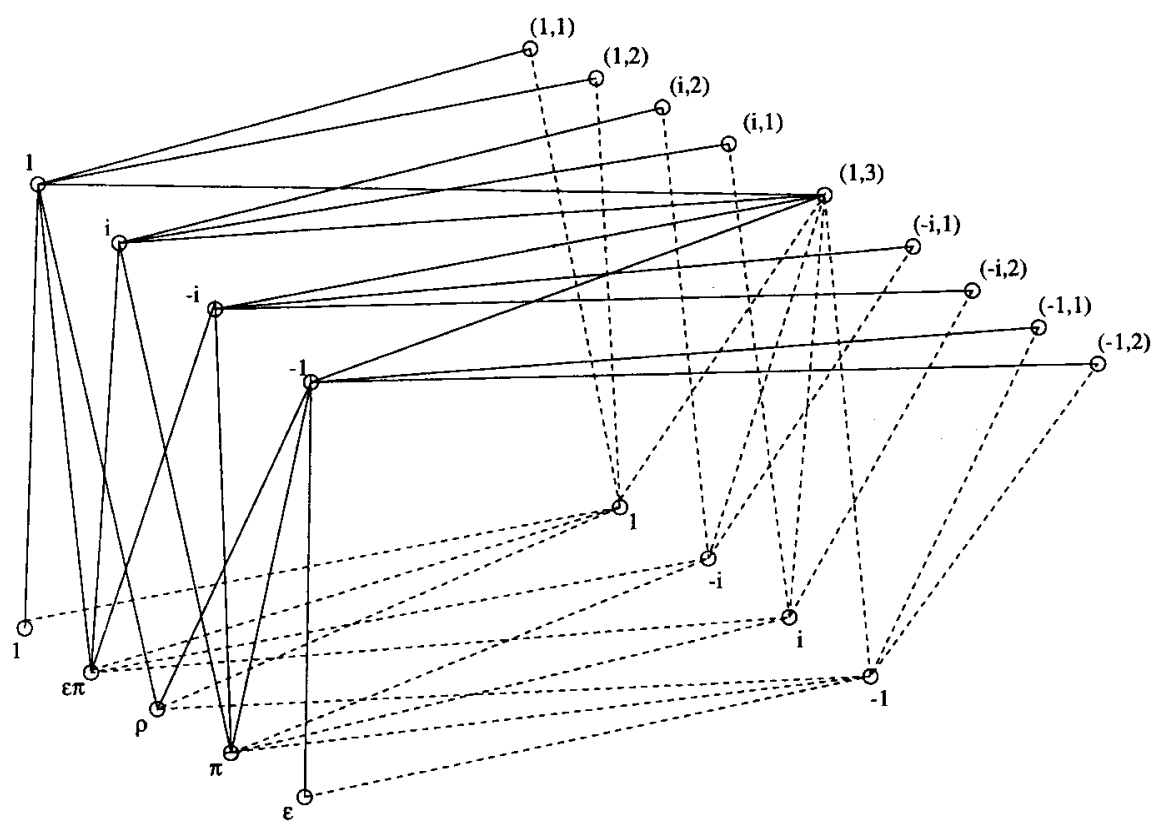

Figure 1: The cells for the subgroup $H$

where $\left\{M_{n}\right\}$ denotes the usual tower of Jones' basic construction. The inclusions of these algebras are described by four Bratteli diagrams; this grand Bratteli diagram is what is shown in Figures 1 and 2 (for the case $L=H$ and $L=K$ respectively. The idea is that the vertices in the north-east (resp., north-west, resp., south-west, resp., south-east) label the minimal central projections in $N^{\prime} \cap M_{2 n+1}$ (resp., $N^{\prime} \cap M_{2 n}$, resp., $M^{\prime} \cap M_{2 n}$, resp., $\left.M^{\prime} \cap M_{2 n+1}\right)$, and the graph contains the data of all the four inclusions. 


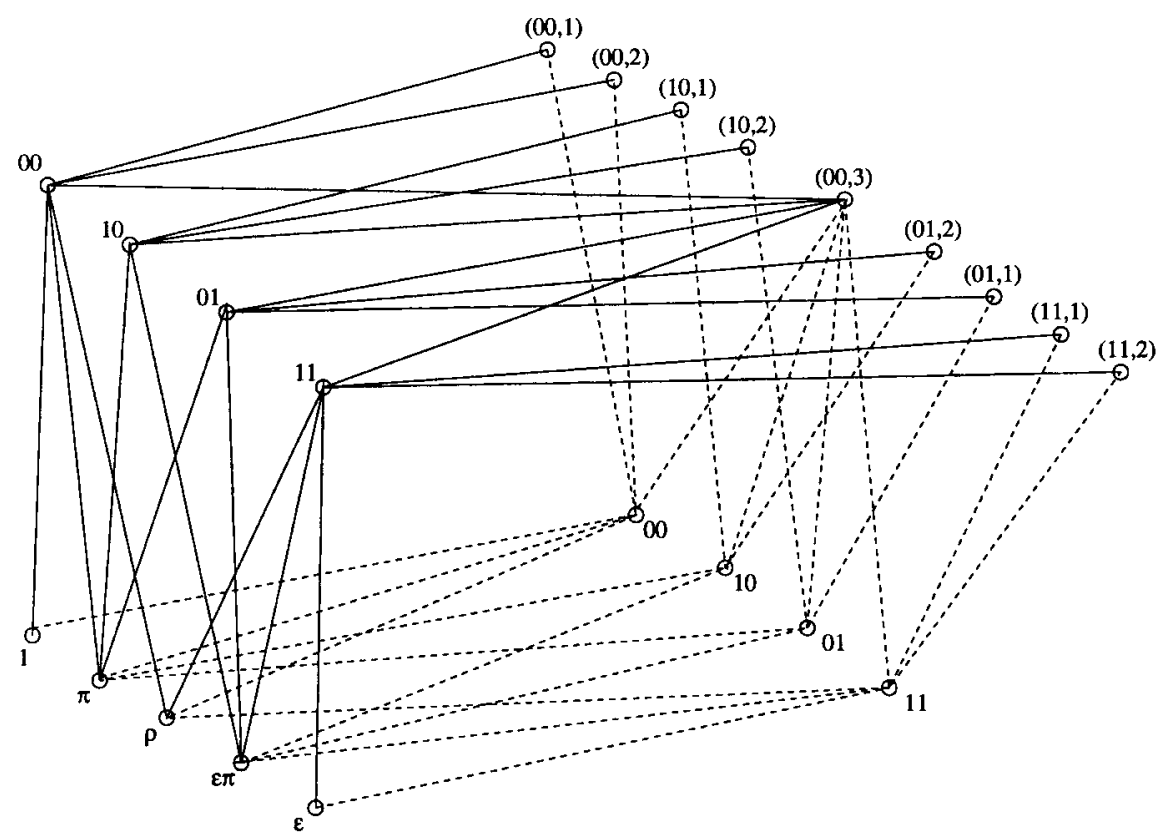

Figure 2: The cells for the subgroup $K$

Next, notice the following pleasant feature of the 'multiplicity data' for restriction between these various groups and subgroups - see Figures 1 and 2: if $G_{1} \subset G_{2}$ is any one of the inclusions $H^{t} \subset H, K^{t} \subset K, H \subset G$ or $K \subset G$, and if $\chi_{i} \in \hat{G}_{i}, i=1,2$, then $\left\langle\operatorname{Res}_{G_{2} \downarrow G_{1}} \chi_{2}, \chi_{1}\right\rangle$ is either 0 or 1 . Consequently, if this multiplicity is 1 , then there is, up to multiplying by a complex scalar of unit modulus, a unique isometric $G_{1}$-linear map from $V_{\chi_{1}}$ into $V_{\chi_{2}}$.

Using a natural blend of the notation of Proposition 14 and the notation used in Figures 1 and 2, we explicitly list out, below, a specification of all pertinent (i.e., non-zero) 'intertwiners':

The intertwiners for the subfactor $(R \times H \subset R \times G)$ :

$$
\begin{array}{rll}
C((1,1), 1) & =C(1,2), 1) & =[1] ; \\
C((i, 2), i) & =C((i, 1), i)=[1] ; \\
C((-i, 1),-i) & =C((-i, 2),-i)=[1] ; \\
C((-1,1),-1) & =C((-1,2),-1)=[1] ; \\
C((1,3), 1)=C((1,3), i) & =C((1,3),-i)=C((1,3),-1)=[1] .
\end{array}
$$




$$
\begin{aligned}
& D(1,1)=D(-1, \epsilon)=[1] \\
& D(1, \epsilon \pi)=D(-1, \pi)=\frac{1}{2}\left[\begin{array}{lllll}
1 & -1 & 1 & -1
\end{array}\right] ; \\
& D(i, \epsilon \pi)=D(-i, \pi)=\frac{1}{2}\left[\begin{array}{llll}
1 & -i & -1 & i
\end{array}\right] ; \\
& D(-i, \epsilon \pi)=D(i, \pi)=\frac{1}{2}\left[\begin{array}{llll}
1 & i & -1 & -i
\end{array}\right] \text {; } \\
& D(1, \rho)=\frac{1}{\sqrt{6}}\left[\begin{array}{lll}
1 & -2 & 1
\end{array}\right] ; \\
& D(-1, \rho)=\frac{1}{\sqrt{2}}\left[\begin{array}{lll}
1 & 0 & -1
\end{array}\right] \text {. } \\
& E(1,1)=E(\epsilon,-1)=[1] \\
& E(\epsilon \pi, 1)=E(\pi,-1)=\frac{1}{2}\left[\begin{array}{c}
1 \\
-1 \\
1 \\
-1
\end{array}\right] \\
& E(\epsilon \pi, i)=E(\pi,-i)=\frac{1}{2}\left[\begin{array}{c}
1 \\
i \\
-1 \\
-i
\end{array}\right] \\
& E(\epsilon \pi,-i)=E(\pi, i)=\frac{1}{2}\left[\begin{array}{c}
1 \\
-i \\
-1 \\
i
\end{array}\right] \\
& E(\rho, 1)=\frac{1}{\sqrt{6}}\left[\begin{array}{c}
1 \\
-2 \\
1
\end{array}\right] \\
& E(\rho,-1)=\frac{1}{\sqrt{2}}\left[\begin{array}{c}
1 \\
0 \\
-1
\end{array}\right] \text {; } \\
& F(1,(1,1))=F(1,(1,2))=[1] ; \\
& F(-i,(i, 2))=F(i,(i, 1))=[1] ; \\
& F(-i,(-i, 1))=F(i,(-i, 2))=[1] ; \\
& F(-1,(-1,1))=F(-1,(-1,2))=[1] \\
& F(1,(1,3))=F(i,(1,3))=F(-i,(1,3))=F(-1,(1,3))=[1] .
\end{aligned}
$$

The intertwiners for the subfactor $(R \times K \subset R \times G)$ : 


$$
\begin{aligned}
& C((00,1), 00)=C(00,2), 00)=[1] ; \\
& C((10,1), 10)=C((01,1), 01)=[i] \\
& C((10,2), 10)=C((01,2), 01)=[-i] ; \\
& C((11,1), 11)=C((11,2), 11)=[1] \\
& C((00,3), 00)=C((00,3), 10)=C((00,3), 01)=C((00,3), 11)=[1] . \\
& D(00,1)=D(11, \epsilon)=[1] \\
& D(10, \epsilon \pi)=D(01, \pi)=\frac{1}{\sqrt{2}}\left[\begin{array}{lllll}
0 & 0 & -i & i
\end{array}\right] ; \\
& D(01, \epsilon \pi)=D(10, \pi)=\frac{1}{\sqrt{2}}\left[\begin{array}{cccc}
1 & -1 & 0 & 0
\end{array}\right] \text {; } \\
& D(11, \epsilon \pi)=D(00, \pi)=\frac{1}{\sqrt{8}}[(-1-i) \quad(-1-i) \quad(1+i) \quad(1+i)] \text {. } \\
& D(00, \rho)=\frac{1}{\sqrt{6}}\left[\begin{array}{lll}
1 & 1 & -2
\end{array}\right] ; \\
& D(11, \rho)=\frac{1}{\sqrt{2}}\left[\begin{array}{lll}
-1 & 1 & 0
\end{array}\right] ; \\
& \begin{array}{l}
E(1,00)=E(\epsilon, 11)=c \\
E(\epsilon \pi, 10)=E(\pi, 01)=\frac{[1] ;}{\sqrt{2}}\left[\begin{array}{c}
0 \\
0 \\
-i \\
i
\end{array}\right]
\end{array} \\
& E(\epsilon \pi, 01)=E(\pi, 10)=\frac{1}{\sqrt{2}}\left[\begin{array}{c}
1 \\
-1 \\
0 \\
0
\end{array}\right] \\
& E(\epsilon \pi, 11)=E(\pi, 00)=\frac{1}{\sqrt{8}}\left[\begin{array}{c}
-1+i \\
-1+i \\
1-i \\
1-i
\end{array}\right] \\
& E(\rho, 00)=\frac{1}{\sqrt{6}}\left[\begin{array}{c}
1 \\
1 \\
-2
\end{array}\right] \\
& E(\rho, 11)=\frac{1}{\sqrt{2}}\left[\begin{array}{c}
-1 \\
1 \\
0
\end{array}\right] \\
& F(00,(00,1))=F(00,(00,2))=[1] ; \\
& F(10,(10,1))=F(10,(01,2))=[1] ; \\
& F(01,(10,2))=F(01,(01,1))=[1] ; \\
& F(11,(11,1))=F(11,(11,2))=[1]
\end{aligned}
$$




$$
F(00,(00,3))=F(10,(00,3))=F(01,(00,3))=F(11,(00,3))=[1] .
$$

We have chosen the intertwiners as above with some care; certain numbers of unit modulus have been specifically chosen and 'standard' multipliers have been multiplied by these numbers, in order that the connections for the two subfactors turn out to be identical - after we identify the two sets of bimodules in accordance with the prescription forced by Figures 1 and 2. If the connections are the same, then the 'canonical commuting squares' of the two subfactors coincide and the subfactors are conjugate, by [6] and [8].

We shall display the connections using the notation discussed in the paragraph preceding Proposition 17. In writing out the connections, we shall employ the following conventions: for either subgroup-subfactor, we will list out the several possible unitary matrices of the form $W((\chi, t), \zeta)$ for all $\zeta \in \hat{G}$ and for $1 \leq t \leq 3$ and $\chi$ a 1 -dimensional character of $L^{t}$, where $L$ is $H$ or $K$ depending on which subfactor we are looking at. In either case, $(\chi, t)$ will label one of the vertices in the north-east corner, while $\zeta$ will label one of the vertices in the south-west corner of the corresponding figure ( 1 for $H$, and 2 for $K)$. The rows (rep., columns) of the matrix $W((\chi, t), \zeta)$ will be indexed by vertices in the north-west (resp., south-east) corner which are simultaneously a neighbour of $(\chi, t)$ as well as of $\zeta$.

We first display all those $W((\chi, t), \zeta)$ which are $1 \times 1$ matrices; for instance, the remarks of the preceding paragraph say that the matrix $W((i, 2), \pi)$ has its unique row (resp., column) indexed by the vertex labelled $i$ (resp., $-i$ ) which is in the north-west (resp., south-east) corner of Figure 1.

$$
\begin{aligned}
& W((1,1), 1)=W((00,1), 1)=[1] ; \\
& W((1,1), \epsilon \pi)=W((00,1), \pi)=[1] ; \\
& W((1,1), \rho)=W((00,1), \rho)=[1] ; \\
& W((1,2), 1)=W((00,2), 1)=[1] ; \\
& W((1,2), \epsilon \pi)=W((00,2), \pi)=[-1] ; \\
& W((1,2), \rho)=W((00,2), \rho)=[1] ; \\
& W((i, 2), \epsilon \pi)=W((10,1), \pi)=[i] ; \\
& W((i, 2), \pi)=W((10,1), \epsilon \pi)=[-i] ; \\
& W((i, 1), \epsilon \pi)=W((10,2), \pi)=[1] ; \\
& W((i, 1), \pi)=W((10,2), \epsilon \pi)=[1] ; \\
& W((-i, 1), \epsilon \pi)=W((01,2), \pi)=[1] ; \\
& W((-i, 1), \pi)=W((01,2), \epsilon \pi)=[1] ;
\end{aligned}
$$




$$
\begin{aligned}
& W((-i, 2), \epsilon \pi)=W((01,1), \pi)=[-i] ; \\
& W((-i, 2), \pi)=W((01,1), \epsilon \pi)=[i] ; \\
& W((-1,1), \rho)=W((11,1), \rho)=[1] ; \\
& W((-1,1), \pi)=W((11,1), \epsilon \pi)=[1] ; \\
& W((-1,1), \epsilon)=W((11,1), \epsilon)=[1] ; \\
& W((-1,2), \rho)=W((11,2), \rho)=[1] ; \\
& W((-1,2), \pi)=W((11,2), \epsilon \pi)=[-1] ; \\
& W((-1,2), \epsilon)=W((11,2), \epsilon)=[1] \text {. }
\end{aligned}
$$

In displaying those remaining $W((\chi, t), \zeta)$ 's which are unitary matrices with more than one row and column, we shall adopt the following convention: we shall 'border' the matrix with an extra row and column (at the right and bottom respectively) whose entry, which will appear inside parentheses, will indicate the vertex labelling the relevant row or column. The 'non-trivial' matrices in the connection are:

$$
\begin{aligned}
& W((1,3), \epsilon \pi)=\frac{1}{2}\left[\begin{array}{ccc|c}
0 & -1-i & -1+i & (1) \\
1-i & -1 & -i & (i) \\
1+i & i & -1 & (-i) \\
\hline(1) & (-i) & (i) &
\end{array}\right] \\
& W((00,3), \pi)=\frac{1}{2}\left[\begin{array}{ccc|c}
0 & -1-i & -1+i & (00) \\
1-i & -1 & -i & (10) \\
1+i & i & -1 & (01) \\
\hline(00) & (10) & (01) &
\end{array}\right] \\
& W((1,3), \rho)=\frac{1}{2}\left[\begin{array}{cc|c}
-1 & -\sqrt{3} & (1) \\
\sqrt{3} & -1 & (-1) \\
\hline(1) & (-1) &
\end{array}\right], \\
& W((00,3), \rho)=\frac{1}{2}\left[\begin{array}{cc|c}
-1 & -\sqrt{3} & (00) \\
\sqrt{3} & -1 & (11) \\
\hline(00) & (11) &
\end{array}\right] \\
& W((1,3), \pi)=\frac{1}{2}\left[\begin{array}{ccc|c}
-1 & i & 1+i & (i) \\
-i & -1 & 1-i & (-i) \\
-1+i & -1-i & 0 & (-1) \\
\hline(-i) & (i) & (-1) &
\end{array}\right]
\end{aligned}
$$




$$
W((00,3), \epsilon \pi)=\frac{1}{2}\left[\begin{array}{ccc|c}
-1 & i & 1+i & (10) \\
-i & -1 & 1-i & (01) \\
-1+i & -1-i & 0 & (11) \\
\hline(10) & (01) & (11) &
\end{array}\right]
$$

Thus, we see that the two commuting squares become identical when we make the following identifications:

$$
\begin{aligned}
& (1,1) \quad(00,1) \\
& (1,2) \quad(00,2) \\
& (i, 2) \quad(10,1) \\
& (i, 1) \quad(10,2) \\
& \mathscr{G}(N, N):(1,3) \quad(00,3) \\
& (-i, 1) \quad(01,2) \\
& (-i, 2) \quad(01,1) \\
& (-1,1) \quad(11,1) \\
& (-1,2) \quad(11,2) \\
& \mathscr{G}(N, M): \begin{array}{cc}
1 & 00 \\
i & 10 \\
-i & 01 \\
-1 & 11
\end{array} \\
& \mathscr{G}(M, N): \begin{array}{cc}
1 & 00 \\
-i & 10 \\
i & 01 \\
-1 & 11
\end{array} \\
& \begin{array}{cc}
\text { for } H \text { for } K \\
\hline 1 & 1
\end{array} \\
& \mathscr{G}(M, M): \begin{array}{cc}
\epsilon \pi & \pi \\
\rho & \rho
\end{array} . \\
& \pi \quad \epsilon \pi \\
& \epsilon \quad \epsilon
\end{aligned}
$$

REMARK 19. The above counter-example to Thomsen's conjecture is 'smallest possible', in the following sense: suppose $H_{i}$ is a subgroup of a finite group $G_{i}$ such that $H_{i}$ contains no non-trivial normal subgroup of $G_{i}$, for $i=1,2$, and $\left[G_{1}: H_{1}\right]=\left[G_{2}: H_{2}\right] \leq 6$, and suppose the subfactors $\left(R \times H_{i} \subset R \times G_{i}\right)$ are isomorphic but the subgroups $\left(H_{i} \subset G_{i}\right)$ are non-isomorphic; then after re-labelling, if necessary, we may deduce that $\left(H_{1} \subset G_{1}\right) \cong\left(H \subset S_{4}\right)$ and $\left(H_{2} \subset G_{2}\right) \cong\left(K \subset S_{4}\right)$. (The way we verified this was with the help of a list of transitive group actions on small sets that we found in [1].) 
In conclusion, we raise the following natural questions:

Questions:

(1) Is there a more transparent group-theoretic description of what it takes for two pairs $H_{i} \subset G_{i}$ to satisfy:

(a) $H_{i}$ contains no non-trivial normal subgroup of $G_{i}$, and

(b) $\left(R \times H_{1} \subset R \times G_{1}\right) \cong\left(R \times H_{2} \subset R \times G_{2}\right)$ ?

(Or, equivalently, is there a non-computational proof of the fact that the counter-example given here to Thomsen's question is indeed a counter-example?)

(2) What is the answer to Thomsen's question, if one requires that the subgroup $H$ is, in addition to containing no non-normal subgroup of $G$, also a maximal subgroup of $G$ (in the sense that there are no non-trivial intermediate subgroups (equivalently, subfactors)?

(3) What are some more examples of the sort described in $\S 6$ ?

\section{REFERENCES}

1. G. Butler and J. McKay, The transitive groups of degree up to eleven, Comm. Alg. 11 (1983), 863-911.

2. V.F.R. Jones and V.S. Sunder, Introduction to Subfactors, London Math. Soc. Lecture Note Ser. 234, 1997

3. H. Kosaki, A. Munemasa, and S. Yamagami, On fusion algebras associated to finite group actions, Pac. J. Math. 177 (1997), 269-290.

4. H. Kosaki and S. Yamagami, Irreducible bimodules associated with crossed product algebras, Internat. J. Math. 3 (1992), 661-676.

5. A. Ocneanu, Quantized groups, string algebras and Galois theory for algebras, Operator Algebras and Applications, Vol. 2, London Math. Soc. Lecture Note Ser. 136, 1988.

6. A. Ocneanu, (Lecture Notes written by Y. Kawahigashi), Quantum symmetry, differential geometry of finite graphs, and classification of subfactors, Univ. of Tokyo Seminar Notes, 1990.

7. S. Popa, Classification of amenable subfactors of type II, Acta Math. 172 (1994), 163-255.

8. S. Popa, Classification of subfactors: the reduction to commuting squares, Invent. Math. 101 (1990), 19-43.

9. K. Thomsen, Factors and subfactors from ergodic theory, talk at Madras Conference, 1997. 